\title{
Some recent applications of the barycenter method in geometry
}

\author{
Christopher Connell and Benson Farb * \\ Department of Mathematics \\ University of Chicago
}

July 26, 2002

\section{Contents}

1 The Entropy Rigidity Conjecture 3

1.1 The main conjecture . . . . . . . . . . . . . 3

1.2 Progress . . . . . . . . . . . . . . . . 5

1.3 The Besson-Courtois-Gallot map . . . . . . . . . . . 6

1.4 The degree theorem . . . . . . . . . . . . . . 7

1.5 A related conjecture . . . . . . . . . . . . . . 9

1.6 Some consequences of the Degree Theorem . . . . . . . . 9

2 The Douady-Earle-Besson-Courtois-Gallot map 11

2.1 The construction . . . . . . . . . . . . . . . 11

2.2 Patterson-Sullivan measures . . . . . . . . . . . . . . . 12

2.3 The barycenter functional . . . . . . . . . . . . . . 13

3 Bounding the Jacobian 15

3.1 Existence of some bound . . . . . . . . . . . . . 15

3.2 The perfect bound in rank one . . . . . . . . . 18

4 Negative results in various settings 18

4.1 Finsler metrics . . . . . . . . . . . . . . . . . . . . . . . . . .

4.2 Magnetic field flows . . . . . . . . . . . . . . 21

4.3 Metric entropy . . . . . . . . . . . . . . . . 22

${ }^{*}$ Both authors are supported in part by the NSF. This paper grew out of talks given by the first and second authors at the Cornell and Georgia Topology conferences, respectively. 
5 Positive results in various settings $\quad 22$

5.1 Finsler metrics . . . . . . . . . . . . . . . . 22

5.2 Foliations . . . . . . . . . . . . . . . 23

5.3 Finite volume manifolds . . . . . . . . . . . . . . 25

5.4 Quasifuchsian representations . . . . . . . . . . . 27

5.5 Alexandroff Spaces . . . . . . . . . . . . . . . 27

5.5.1 Convex Cores . . . . . . . . . . . . . 29

5.5.2 Application to Cone Manifolds . . . . . . . . . 30

6 Cautionary examples $\quad 31$

$6.1 \quad$ An example in $\mathbf{H}^{2} \times \mathbf{H}^{2} \ldots \ldots \ldots \ldots \ldots$

6.2 An example in $\mathrm{SL}_{3}(\mathbf{R}) / \mathrm{SO}_{3}(\mathbf{R}) \ldots \ldots \ldots . \ldots . \ldots . \ldots 33$

6.3 An example in $\mathrm{SL}_{4}(\mathbf{R}) / \mathrm{SO}_{4}(\mathbf{R}) \ldots \ldots \ldots . \ldots . \ldots 34$ 


\section{The Entropy Rigidity Conjecture}

In this paper we describe some recent applications of the barycenter method in geometry. This method was first used by Douady-Earle and later greatly extended by Besson-Courtois-Gallot in their solution of a number of longstanding problems, in particular in their proof of entropy rigidity for closed, negatively curved locally symmetric manifolds. Since there are already a number of surveys describing this work (see [BCG2, BCG3, Pa, Ga]), we will concentrate here only on advances that have occurred after these surveys appeared. While most of this paper is a report on results appearing in other papers, some of the material here is new (e.g. Proposition 12 and the examples in Section 6).

\subsection{The main conjecture}

The volume entropy of a closed Riemannian $n$-manifold $(M, g)$, denoted by $h(g)$, is defined to be

$$
h(g)=\lim _{R \rightarrow \infty} \frac{1}{R} \log (\operatorname{Vol}(B(x, R)))
$$

where $B(x, R)$ is the ball of radius $R$ around a fixed point $x$ in the universal cover $X$. The number $h(g)$ is independent of the choice of $x$, and equals the topological entropy of the geodesic flow on $(M, g)$ when the curvature $K(g)$ satisfies $K(g) \leq 0$ (see [Ma]). Note that while neither the volume $\operatorname{Vol}(M, g)$ nor the entropy $h(g)$ is invariant under scaling the metric $g$, the normalized entropy

$$
\operatorname{ent}(g)=h(g)^{n} \operatorname{Vol}(M, g)
$$

is scale invariant.

Now let $M$ be a closed $n$-manifold that admits a locally symmetric Riemannian metric $g_{l o c}$ of nonpositive sectional curvature. When $\left(M, g_{l o c}\right)$ is not locally isometric to a product then $g_{l o c}$ is unique up to homothety (i.e. multiplying the metric by a number). When $M$ is locally a product, one may show using Lagrange multipliers that there is a unique (up to homothety) locally symmetric metric on $M$ which minimizes ent $(g)$; see [CF1]. Henceforth we will abuse notation and denote this metric by $g_{l o c}$, and call it the locally symmetric metric.

The Entropy Rigidity Conjecture, stated in various forms by Katok, Gromov, and Besson-Courtois-Gallot (see, e.g., [BCG2], Open Question 5), has two components. The purely metric component posits that for most $M$, the metric $g_{l o c}$ minimizes the functional ent $(g)$ over the space of all Riemannian 
metrics on $M$, and in fact uniquely minimizes ent $(g)$ (up to homothety). This property would characterize the locally symmetric metric by essentially a single number. The topological component of the conjecture is an extension of this statement from manifolds to maps.

Conjecture 1 (Entropy Rigidity Conjecture). Let $M$ be a closed manifold which admits a locally symmetric Riemannian metric $g_{l o c}$ with nonpositive sectional curvature. Assume that $\left(M, g_{l o c}\right)$ has no local factors isometric to $\mathbf{R}$. Let $(N, g)$ be any closed Riemannian manifold, and let $f: N \longrightarrow M$ be any continuous map. Then

$$
\operatorname{ent}(N, g) \geq|\operatorname{deg} f| \operatorname{ent}\left(M, g_{l o c}\right)
$$

with equality if and only if $f$ is homothetic to a Riemannian covering.

\section{Remarks:}

1. The case when $f$ is a homeomorphism, or even the identity map, gives that $g_{l o c}$ uniquely minimizes ent $(g)$.

2. Easy examples show that the restrictions to $M$ with no local $\mathbf{R}$ factors is necessary.

3. Conjecture 1 easily implies Mostow Rigidity; the argument is the same as in the case when $M$ is negatively curved, which is given in [BCG2].

Conjecture 1 may be extended to a variety of other contexts, for example to finite volume metrics, Finsler metrics, foliations with locally symmetric leaves, contact flows, and magnetic flows. To be more precise, the table below shows some natural choices for the volume and entropy in various metric settings. In the table we have adopted the following conventions:

- $\theta$ represents a contact 1 -form

- $h_{m}$ is metric entropy, i.e. the measure theoretic entropy for the Liouville measure

- $h_{\text {top }}$ represents the topological entropy of the geodesic flow

- $h_{\mathrm{vol}}$ is the volume growth entropy

- $\operatorname{dim}_{\mathcal{H}}(\Lambda(\Gamma))$ is the Hausdorff dimension of the limit set $\Lambda(\Gamma)$ of a discrete group $\Gamma$ of hyperbolic isometries

- $M=Y / \Gamma$

These extensions are discussed in greater depth in Sections 4 and 5 . 


\begin{tabular}{|l|l|l|}
\hline Setting & Volume $\operatorname{Vol}(M)$ & entropy $h(d)$ \\
\hline \hline $\begin{array}{l}\text { Riemannian metrics } \\
\text { on closed } M\end{array}$ & Riem. volume & $h_{\mathrm{vol}}$ \\
\hline $\begin{array}{l}\text { Finite vol. metrics } \\
\text { on noncompact } M\end{array}$ & Riem. volume & $\inf \left\{s: \int_{Y} e^{-s d(p, y)} d g(y)<\infty\right\}$ \\
\hline Contact flows & $\int_{M} \theta \wedge(d \theta)^{n-1}$ & $h_{\text {top }}$ \\
\hline Finsler metrics & Finsler Volume & $\mathrm{h}_{\mathrm{top}}$ \\
\hline Magnetic field flows & $\begin{array}{l}\text { Euler-Lagrange } \\
\text { Volume }\end{array}$ & $h_{\mathrm{top}}$ \\
\hline Foliations on $M$ & $\begin{array}{l}d \text { Vol }_{g_{L}} \times d \operatorname{Vol}(L): \\
\text { leafwise volume } \\
\text { transverse volume }\end{array}$ & $\left(\frac{1}{\operatorname{Vol}(M)} \int_{M} h\left(g_{L}\right)^{n} d \operatorname{Vol}\right)^{1 / n}$ \\
\hline $\begin{array}{l}\text { Geometrically } \\
\text { finite } M\end{array}$ & \begin{tabular}{l} 
Volume of core \\
\hline
\end{tabular} & $\operatorname{dim}_{\mathcal{H}}(\Lambda(\Gamma))$ \\
\hline
\end{tabular}

Figure 1: Some natural choices for the volume and entropy in different settings. The functional being minimized is ent $:=h(d)^{n} \operatorname{Vol}(M)$.

\subsection{Progress}

The main evidence for Conjecture 1 is given by the following theorem.

Theorem 2 (Besson-Courtois-Gallot [BCG1]). Conjecture 1 is true when $\left(M, g_{l o c}\right)$ has $\mathbf{R}$-rank one, i.e. when $\left(M, g_{l o c}\right)$ is negatively curved.

This result and its proof have a number of corollaries, including solutions to long-standing problems on geodesic flows, asymptotic harmonicity, and Gromov's Minvol invariant; these are described in [BCG2].

In higher rank very little is known. The following was announced in [BCG2] and later in [BCG3], and was proved in [CF1] (in the finite volume case as well).

Theorem 3. Conjecture 1 is true when $\left(M, g_{l o c}\right)$ is locally (but not necessarily globally) isometric to a product of negatively curved locally symmetric spaces.

It seems that a significantly new idea is needed to prove Conjecture 1. See, however, $\S 1.4$ below. 


\subsection{The Besson-Courtois-Gallot map}

Building on earlier work of Douady-Earle, Besson-Courtois-Gallot constructed a remarkable map $F$ in every homotopy class of maps $f: N \rightarrow M$ from an arbitrary closed manifold $N$ to any closed manifold $M$ of negative sectional curvature. This map is a kind of replacement for, and has several advantages over, the harmonic map in the homotopy class of $f$; in particular, it can often be differentiated explicitly. The map $F$ is a key ingredient in the proof of Theorem 2.

Now suppose that, in addition to being negatively curved, $M$ is also locally symmetric with metric $g_{l o c}$. In this case Besson-Courtois-Gallot were able to give a precise estimate on the Jacobian $\mid$ Jac $F \mid$, proving Theorem 2. To state things precisely, it will be useful to fix a parameter $s>h(g)$, where $g$ is the metric on $N$ and $h(g)$ is the volume growth entropy of $g$. When $\left(M, g_{l o c}\right)$ has $\mathbf{R}$-rank one, that is when it is negatively curved, BessonCourtois-Gallot proved that, given any continuous $f: N \longrightarrow M$, there exists a smooth map $F_{s}: N \longrightarrow M$ homotopic to $f$ with the following two important properties:

Universal Jacobian bound: For all $s>h(g)$ and all $y \in N$ we have:

$$
\left|\operatorname{Jac} F_{s}(y)\right| \leq\left(\frac{s}{h\left(g_{l o c}\right)}\right)^{n}
$$

Infinitesimal rigidity: There is equality in equation (2) at the point $y \in N$ if and only if $D_{y} F_{s}$ is a homothety.

Before explaining how to construct such an $F_{s}$ (which we do in $\S 2$ ), let us deduce some consequences of its existence.

The existence of any $F_{s}$ satisfying the two properties above implies Conjecture 1 by an elementary degree argument, as follows. Since for $s>h(g)$, the map $F_{s}$ is a $C^{1}$ map, we may simply compute:

$$
\begin{aligned}
|\operatorname{deg}(f)| \operatorname{Vol}(M) & =\left|\operatorname{deg}\left(F_{s}\right)\right| \operatorname{Vol}(M) \\
& =\left|\int_{N} F_{s}^{*} d g_{l o c}\right| \\
& \leq \int_{N}\left|\operatorname{Jac} F_{s}\right| d g \\
\leq & \left(\frac{s}{h\left(g_{l o c}\right)}\right)^{n} \operatorname{Vol}(N)
\end{aligned}
$$


Letting $s \longrightarrow h(g)$ gives the inequality in Conjecture 1 . In the case when equality is achieved, after scaling the metric $g$ by the constant $\frac{h(g)}{h\left(g_{l o c}\right)}$, we have $h(g)=h\left(g_{l o c}\right)$ and $\operatorname{Vol}(N)=|\operatorname{deg}(f)| \operatorname{Vol}(M)$.

\subsection{The degree theorem}

It is useful for us to restate the inequality (1) of the Entropy Rigidity Conjecture as

$$
\operatorname{deg}(f) \leq C \frac{\operatorname{Vol}(N)}{\operatorname{Vol}(M)}
$$

where $C=\left(\frac{h(g)}{h\left(g_{0}\right)}\right)^{n}$. In particular, proving the inequality (3) with this precise value of $C$ gives that the locally symmetric metric $g_{l o c}$ minimizes ent $(g)$ among all metrics $g$ on $N$.

When the inequality (3) holds for some (universal) $C$, perhaps bigger than $\left(\frac{h(g)}{h\left(g_{0}\right)}\right)^{n}$, then the degree argument given in $\S 1.3$ gives a universal bound on the degree of any map of any manifold into $N$. This can be viewed as the topological part of Conjecture 1 . When $N$ is negatively curved, Gromov proved this fundamental relationship between degree and volume in his paper [Gr] (see his Volume Comparison Theorem).

We note that by universal we mean that $C$ depends only on the dimension of the manifolds $M, N$ and on their smallest Ricci curvatures. Scaling the metrics shows that these dependencies are necessary.

When the sectional curvatures of $N$ are not necessarily negative but only nonpositive, the situation is more complicated. Since the $n$-dimensional torus has flat metrics and also has self-maps of arbitrary degree, one needs at least to assume that $N$ has no local $\mathbf{R}$ factors in order that (3) be true. Using the barycenter technique (see below), we proved the following in [CF2].

Theorem 4 (The Degree Theorem). Let $M$ be a closed, locally symmetric n-manifold with nonpositive sectional curvatures. Assume that $M$ has no local direct factors locally isometric to $\mathbf{R}, \mathbf{H}^{2}$, or $\mathrm{SL}_{3}(\mathbf{R}) / \mathrm{SO}_{3}(\mathbf{R})$. Then for any closed Riemannian manifold $N$ and any continuous map $f: N \longrightarrow M$,

$$
\operatorname{deg}(f) \leq C \frac{\operatorname{Vol}(N)}{\operatorname{Vol}(M)}
$$

where $C>0$ depends only on $n$ and the smallest Ricci curvatures of $N$ and $M$.

While the "no local $\mathbf{R}$ factors" assumption is necessary, we believe that Theorem 4 should hold in all other cases. The case when $M$ is locally 
modelled on $\mathrm{SL}_{n}(\mathbf{R}) / S O_{n}(\mathbf{R}), n \geq 2$ also follows from work of Savage [Sa]; see $\S 1.5$ below.

Theorem 4 follows immediately from the degree argument given above (in $\S 1.3$ ) together with the following theorem, which is the main theorem of [CF2], and which we believe is of independent interest.

Theorem 5 (Universal Jacobian bound). Any continuous map $f: N \rightarrow$ $M$ between closed $n$-manifolds, with $M$ nonpositively curved and locally symmetric (barring the exceptions of Theorem 4), is homotopic to a $C^{1}$ map $F$ with

$$
|\operatorname{Jac} F| \leq C
$$

for some constant $C$ depending only on $n$ and on the smallest Ricci curvatures of $M$ and $N$.

The map $F$ is the extension to nonpositively curved locally symmetric manifolds of the Besson-Courtois-Gallot map; we discuss this below.

As we will discuss in $\S 3$, the universal bound for $|\mathrm{Jac} F|$ is obtained by reducing the problem to a minimization problem over a space of measures on a certain Lie group. The Lie group appears because the natural boundary attached to symmetric spaces may be described algebraically. For general nonpositively curved manifolds no such description is available. But the following still seems possible.

Conjecture 6 (Degree Conjecture in nonpositive curvature). Let $M$ be a closed n-manifold with nonpositive sectional curvature, negative Ricci curvature, and no local $\mathbf{R}$ factors. Then for any closed Riemannian manifold $N$ and any continuous map $f: N \longrightarrow M$,

$$
\operatorname{deg}(f) \leq C \frac{\operatorname{Vol}(N)}{\operatorname{Vol}(M)}
$$

where $C>0$ depends only on $n$ and the smallest Ricci curvatures of $N$ and $M$.

The evidence for Conjecture 6 is that it is true when $M$ has negative sectional curvature (Gromov [Gr]), and (almost always) when $M$ is locally symmetric (by Theorem 4, since for locally symmetric manifolds of nonpositive sectional curvature, no local $\mathbf{R}$ factors implies negative Ricci curvature ). The "negative Ricci curvature" hypothesis in Conjecture 6 is necessary, as the following example (see [BGS]) shows. 
Example 7. Let $S$ be a negatively curved surface with one boundary component $C$. While keeping $S$ nonpositively curved we may smooth a neighborhood of $C$ into a cylinder which is metrically $[0, \epsilon) \times S^{1}$, where $C$ is identified to $\{0\} \times S^{1}$. Call the resulting surface $T$.

Now take two copies $X_{1}$ and $X_{2}$ of the manifold $T \times S^{1}$ and glue $X_{1}$ to $X_{2}$ by identifying $[0, \epsilon / 3] \times S^{1} \times S^{1} \subset X_{1}$ and $[0, \epsilon / 3] \times S^{1} \times S^{1} \subset X_{2}$ by the map $(t, x, y) \mapsto(\epsilon / 3-t, y, x)$. The resulting 3 -manifold $X$ has no flats, but has some zero curvature at every point and even has an open subset of zero curvature, namely the subset $Z \subset X$ formed from the union of the images of $[0, \epsilon) \times S^{1}$ in each $X_{i}$.

We note that the two overlapping pieces, $\left(X_{1} \backslash Z\right) \cup(\epsilon / 2, \epsilon) \times S^{1} \times S^{1}$ and $\left(X_{1} \backslash Z\right) \cup(\epsilon / 2, \epsilon) \times S^{1} \times S^{1}$, each carry an $S^{1}$ action that is coherent with a torus action on $Z$ which acts the same in each factor. Therefore, the whole of $X$ carries an $F$-structure in the sense of Cheeger and Gromov [CG]. This implies via $[\mathrm{PaPe}, \mathrm{CG}]$ that $\operatorname{Minvol}(X)=0$ (see below for the definition of $\operatorname{Minvol}(X))$; in particular, the conclusion of Conjecture 6 cannot hold for $X$, even when $f$ is the identity map.

Note that $X$ has no local direct factors locally isomorphic to $\mathbf{R}$, but that $X$ does have some points with some direction of zero Ricci curvature.

\subsection{A related conjecture}

Gromov has made the following:

Conjecture 8 (Positivity of Gromov norm). The Gromov norm of a closed, nonpositively curved, locally symmetric manifold $M$ with no local $\mathbf{R}$ factors is positive.

Conjecture 8 was proven by Savage $[\mathrm{Sa}$ ] when $M$ is locally isometric to $\mathrm{SL}_{n}(\mathbf{R}) / S O_{n}(\mathbf{R})$. While we do not see how positivity of Gromov norm for $M$ directly implies the inequality (3), Savage's proof does imply this inequality. The key point is that Savage proves that any simplex in $M$ can be "straightened" to a simplex with universally bounded volume. This should hold for all symmetric spaces of noncompact type, but as far as we know this is still an open question.

\subsection{Some consequences of the Degree Theorem}

We end this section by recalling some consequences, given in [CF2], of Theorem 4 . 
The Minvol invariant. One of the basic invariants associated to a smooth, closed manifold $M$ is its minimal volume:

$$
\operatorname{Minvol}(M):=\inf _{g}\{\operatorname{Vol}(M, g):|K(g)| \leq 1\}
$$

where $g$ ranges over all smooth metrics on $M$ and $K(g)$ denotes the sectional curvature of $g$. The basic questions about $\operatorname{Minvol}(M)$ are: for which $M$ is $\operatorname{Minvol}(M)>0$ ? when is $\operatorname{Minvol}(M)$ realized by some metric $g$ ?

When a closed, nonpositively curved manifold $M$ has a local direct factor locally isometric to $\mathbf{R}$, then $M$ has some finite cover $M^{\prime}$ with $S^{1}$ as a direct factor. This in turn implies that $M^{\prime}$ has positive degree self-maps and that $\operatorname{Minvol}\left(M^{\prime}\right)=0$. On the other hand, in the statement of Theorem 4 we may take $f$ to be the identity map for an $M$ which admits a locally symmetric metric and allow only the metric $g$ on $M$ to vary. Theorem 4 then shows $\operatorname{Minvol}(M)>0$ for locally symmetric $M$, barring the possible exceptional cases listed in Theorem 4. However, positivity was already known more generally, by the following result of Gromov (see also [Sa] for the case of $M$ locally isometric to the symmetric space for $\mathrm{SL}(n, \mathbf{R}))$.

Corollary 9 (Positivity of Minvol). Let $M$ be a closed, locally symmetric manifold with nonpositive curvature and no local $\mathbf{R}$ factors. Then $\operatorname{Minvol}(M)>0$.

When $M$ is real hyperbolic, Besson-Courtois-Gallot [BCG1] proved that $\operatorname{Minvol}(M)$ is uniquely realized by the locally symmetric metric.

Problem 10. Compute $\operatorname{Minvol}(M)$ for all closed $M$ which admit a locally symmetric metric of nonpositive curvature, with no local $\mathbf{R}$ factors. Is $\operatorname{Minvol}(M)$ always realized by the locally symmetric metric? Is it realized uniquely?

Self maps and the co-Hopf property. $\quad \operatorname{As} \operatorname{deg}\left(f^{n}\right)=\operatorname{deg}(f)^{n}$, an immediate corollary of Theorem 4 is the following.

Corollary 11 (Self maps). Let $M$ be a closed, locally symmetric manifold as in Theorem 4. Then $M$ admits no self-maps of degree $>1$. In particular, $\pi_{1}(M)$ is co-Hopfian: every injective endomorphism of $\pi_{1}(M)$ is surjective.

Note that Corollary 11 may also be deduced from Margulis' Superrigidity theorem (for higher rank $M$ ). The co-Hopf property for lattices was first proved by Prasad [Pr]. 


\section{The Douady-Earle-Besson-Courtois-Gallot map}

\subsection{The construction}

In this section we describe the canonical map $F$ discussed above. This construction is due to Douady-Earle for the hyperbolic plane, was extended by Besson-Courtois-Gallot [BCG4] to negatively curved targets, and was extended to symmetric spaces of noncompact type in [CF1, CF2]. In this section we will extend this construction further to nonpositively curved targets with negative Ricci curvature. For background on nonpositively curved manifolds and symmetric spaces, see for example [BGS, Eb].

As above, let $M, N$ be closed, Riemannian $n$-manifolds with $M$ nonpositively curved, and let $f: N \longrightarrow M$ be any continuous map. Denote by $Y$ (resp. $X$ ) the universal cover of $N$ (resp. $M$ ). Denote by $\partial X$ the visual boundary of $X$; that is, the set of equivalence classes of geodesic rays in $X$, endowed with the cone topology. Hence $X \cup \partial X$ is a compactification of $X$ which is homeomorphic to a closed ball; see, e.g. [BGS].

Idea of the main construction. Let $\phi$ denote the lift to universal covers of $f$ with basepoint $\underset{p}{p} \in Y$ (resp. $f(p) \in X)$, i.e. $\phi=\widetilde{f}: Y \longrightarrow X$. We first construct a map $\widetilde{F}: Y \longrightarrow X$ by "averaging" $\phi$ as follows: first embed $Y$ into a space of measures on $Y$, then push forward each measure via $\phi$, then smooth out the measure onto $\partial X$ by convolving with a canonical measure on $\partial X$, and finally take the "barycenter" of the resulting measure. An essential feature of each of these steps is that they are canonical, i.e. they are equivariant with respect to the actions of fundamental groups on each of the spaces involved. It follows from this that $\widetilde{F}$ descends to a map $F: N \longrightarrow M$.

Now to define $\widetilde{F}$ precisely. Actually, it is useful to fix a parameter $s>$ $h(g)$ for which we define a map $\widetilde{F}_{s}$. We denote the Riemannian metric and corresponding volume form on universal cover $Y$ by $g$ and $d g$ respectively.

Let $\mathcal{M}(Z)$ denote the space of probability measures on the set $Z$. Following the method of [BCG1], we define a map $\widetilde{F}_{s}: Y \longrightarrow X$ as a composition

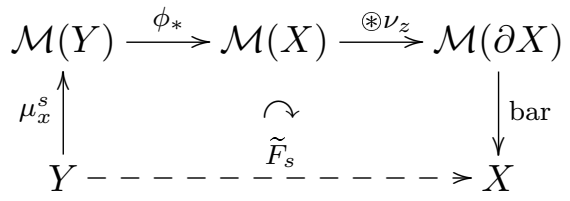

where the individual maps are defined as follows: 
- The inclusion $Y \longrightarrow \mathcal{M}(Y)$, denoted $y \mapsto \mu_{y}^{s}$, is given by localizing the Riemannian volume form $d g$ on $Y$ and normalizing it to be a probability measure; that is, $\mu_{y}^{s}$ is the probability measure on $Y$ in the Lebesgue class with density given by

$$
\frac{d \mu_{y}^{s}}{d g}(z)=\frac{e^{-s d(y, z)}}{\int_{Y} e^{-s d(y, z)} d g}
$$

Note that each $\mu_{y}^{s}, y \in Y$ is well-defined by the choice of $s$.

- The map $\phi_{*}$ is the push forward of measures.

- The symbol $\circledast \nu_{z}$ indicates the operation of convolution with the PattersonSullivan measures $\left\{\nu_{x}\right\}_{x \in X}$ corresponding to $\pi_{1}(M)<\operatorname{Isom}(X)$ (see $\S 2.2)$. In other words, the resulting measure $\sigma_{y}^{s}$ is defined on a Borel set $U \subset \partial X$ by

$$
\sigma_{y}^{s}(U)=\int_{X} \nu_{z}(U) d\left(\phi_{*} \mu_{y}^{s}\right)(z)
$$

Since $\left\|\nu_{z}\right\|=1$, we have

$$
\left\|\sigma_{y}^{s}\right\|=\left\|\mu_{y}^{s}\right\|=1
$$

- The map bar is the barycenter of the measure $\sigma_{y}^{s}$, which is defined as the unique minimum of a certain functional on $X$, depending on the measure (see $§ 2.3$ ). The map bar is not always defined; when it is defined its domain of definition often must be restricted.

We now describe the last two maps in more detail. For full details in the current context we refer the reader to [CF2].

\section{$2.2 \quad$ Patterson-Sullivan measures}

Let $\Gamma$ be a discrete group of isometries of a connected, simply-connected, complete, nonpositively curved manifold $X$ with no Euclidean direct factors. Fix a basepoint $p \in X$.

Generalizing work of Patterson and Sullivan, a number of authors, including Coornaert, Margulis (unpublished), Albuquerque, and Knieper constructed a remarkable family $\left\{\nu_{x}\right\}$ of probability measures on $\partial X$. These measures, called Patterson-Sullivan measures, are meant to encode the density of an orbit $\Gamma \cdot p$ at infinity as viewed from $y \in Y$, giving the PattersonSullivan measure $\nu_{y}$ on $\partial_{\infty} Y$. See $[\mathrm{Al}]$ and $[\mathrm{Kn}]$ for the construction of Patterson-Sullivan measures in nonpositive curvature. 
The key properties of Patterson-Sullivan measures $\nu_{x}$ are:

1. No atoms: Each $\nu_{x}$ has no atoms.

2. Equivariance: $\nu_{\gamma x}=\gamma_{*} \nu_{x}$ for all $\gamma \in \Gamma$.

3. Explicit Radon-Nikodym derivative: For all $x, y \in X$, the measure $\nu_{y}$ is absolutely continuous with respect to $\nu_{x}$. In fact the RadonNikodym derivative is given explicitly by:

$$
\frac{d \nu_{x}}{d \nu_{y}}(\xi)=e^{h(g) B(x, y, \xi)}
$$

where $B(x, y, \xi)$ is the Busemann function on $X$. For points $x, y \in X$ and $\xi \in \partial X$, the function $B: X \times X \times \partial X \rightarrow \mathbf{R}$ is defined by

$$
B(x, y, \xi)=\lim _{t \rightarrow \infty} d_{X}\left(y, \gamma_{\xi}(t)\right)-t
$$

where $\gamma_{\xi}$ is the unique geodesic ray with $\gamma(0)=x$ and $\gamma(\infty)=\xi$.

4. Support(Knieper [Kn], Albuquerque [Al]): Each $\nu_{x}$ is supported on a specific $\Gamma$-orbit in $\partial_{\infty} X$.

The importance of property (3) is that it makes possible a reasonably explicit computation of the Jacobian of the map $F_{s}$; see below.

Property (4) is the most difficult of the properties to prove (see $[\mathrm{Al}]$ for the locally symmetric case and $[\mathrm{Kn}]$ for the geometric rank one case). Its importance is that when $X$ is a symmetric space of noncompact type, the support of each $\nu_{x}$ lies in a specific copy of the Furstenberg boundary $\partial_{F} X$, and thus may be identified algebraically. This allows one to convert a geometry problem into an algebra problem; see $\S 3.1$ below.

\subsection{The barycenter functional}

For a probability measure on $\partial X$, one can try to define its barycenter, or center of mass, as follows. One first defines a map

$$
\mathcal{B}: X \times \mathcal{M}(\partial X) \longrightarrow \mathbf{R}
$$

via

$$
\mathcal{B}(x, \lambda)=\int_{\partial_{\infty} X} B(x, \theta) d \lambda(\theta)
$$


where $B$ is the Busemann function on $X$, based at a fixed basepoint. Since $X$ has nonpositive sectional curvatures, the distance function $d$ on $X$ is convex, and hence so is the Busemann function $B$. It follows that for any fixed measure $\lambda \in \mathcal{M}(\partial X)$, the function $\mathcal{B}(\cdot, \lambda)$ is a convex function on $X$. While $\mathcal{B}(\cdot, \lambda)$ is not in general strictly convex (for example when $X$ is Euclidean, it is in many important cases.

Proposition 12 (strict convexity). Suppose that $X$ has nonpositive sectional curvature, negative Ricci curvature uniformly bounded away from 0 , and no local $\mathbf{R}$ factors. Let $\lambda \in \mathcal{M}(\partial X)$ be a fixed measure. Suppose that either

- $X$ is a symmetric space of noncompact type and $\operatorname{supp}(\lambda)$ is the Furstenberg boundary of $X$, or

- $X$ has geometric rank one and $\operatorname{supp}(\lambda)=\partial X$.

Then the function $\mathcal{B}(\cdot, \lambda)$ is strictly convex on $X$.

We note that in both cases of Proposition 12 the hypotheses are satisfied when $\lambda$ is Patterson-Sullivan measure.

Proof. The case when $X$ is a symmetric space of noncompact type is Proposition 3.1 of [CF2]. Hence we assume that $X$ has geometric rank one and negative Ricci curvature (say bounded by $-a^{2}$ ). We also fix a basepoint $p$ for the Busemann function $B$ on $X$.

As the Busemann $B$ function is convex, the functional

$$
\mathcal{B}(y, \lambda):=\int_{\partial X} B(y, p, \xi) d \lambda(\xi)
$$

is convex, being an integral of convex functions. We must prove that $\mathcal{B}(\cdot, \lambda)$ is strictly convex on $X$.

To see this, we first note that while the Hessian of $B(\cdot, p, \xi)$ is only semidefinite for fixed $\xi$, after taking the integral it becomes positive definite provided that $D_{v} d_{y} B$ does not have a common 0 direction $w \in S_{y} Y$ for each $v \in S_{y} Y$ which lies in the support of $\lambda$. Since the support of $\lambda$ is all of $\partial X$, we must show that the tensors $D_{v} d_{y} B$ do not have a common zero $w$.

Now we have the decomposition

$$
\left(D_{v} d_{y} B\right)^{*}=0 \oplus U(v)
$$

where $U(v)$ is the second fundamental form of the horosphere through $v$. In particular the Ricatti equations imply that $U(v)$ is a semi-definite tensor 
on the subspace of $T_{y} Y$ orthogonal to $v$. Therefore $w$ must lie in $\langle v\rangle$ $\oplus \operatorname{ker} U(v)$ for all $v \in S_{y} Y$, or for all $v \neq \pm w$ we have $w-<w, v>v \in$ $\operatorname{ker} U(v)$.

Consider such a $w \neq v$. Let $J(t)$ be the unique Jacobi field $J(t)$ along the geodesic $v(t)$ with $v^{\prime}(0)=v$ satisfying $J(0)=w$ and $J(t)$ bounded for all $t>0$. Then $J$ also satisfies $J^{\prime}(0)=0$, that is $J(t)$ is parallel along $v$ at $t=0$. In particular the Ricatti equation then implies that $R(v, w, v, w)=0$. Since this is true for all $v \in S_{y} Y$ with $v \neq w$ then $R_{w}:=R(w, \cdot, w, \cdot)$ is the zero tensor, but we assumed that the Ricci curvature $\operatorname{tr} R_{w}$ at $w$ was nonzero, and we are done. $\diamond$

Note that Example 7 on page 9 shows that the negativity condition on the Ricci curvature is necessary.

Suppose that $X$ satisfies the hypotheses of Proposition 12. Since we also know that the strictly convex function $\mathcal{B}(y, \lambda)$ goes to infinity as $x$ goes to infinity we can define:

$$
\begin{gathered}
\text { bar }: \mathcal{M}\left(\partial_{\infty} X\right) \longrightarrow X \\
\operatorname{bar}(\lambda):=\text { unique minimum of } \mathcal{B}(\cdot, \lambda)
\end{gathered}
$$

We can now define $\widetilde{F}: Y \longrightarrow X$ by

$$
\widetilde{F}(x):=\operatorname{bar}\left(\widetilde{f}_{*}\left(\mu_{x}^{s}\right) \circledast \nu_{x}\right)
$$

as described above. From the equivariance properties described above, it follows that $\widetilde{F}$ is equivariant with respect to the homomorphism $f_{*}: \pi_{1}(N) \longrightarrow$ $\pi_{1}(M)$, so that $\widetilde{F}$ descends to a map $F: N \longrightarrow M$.

\section{Bounding the Jacobian}

The power of the map $F$ comes from the fact that one can often obtain explicit estimates on its Jacobian. As we saw in $\S 1.3$, such estimates are the key to all applications of the barycenter method. In this section, we sketch how estimates on $|\operatorname{Jac}(F)|$ have been obtained in some cases.

\subsection{Existence of some bound}

Let $\sigma_{y}^{s}=\widetilde{f}_{*}\left(\mu_{x}^{s}\right) \circledast \nu_{x}$. Now $F_{s}$ is defined by the implicit vector equation:

$$
\int_{\partial_{\infty} X} d B_{\left(F_{s}(y), \theta\right)}(\cdot) d \sigma_{y}^{s}(\theta)=0
$$




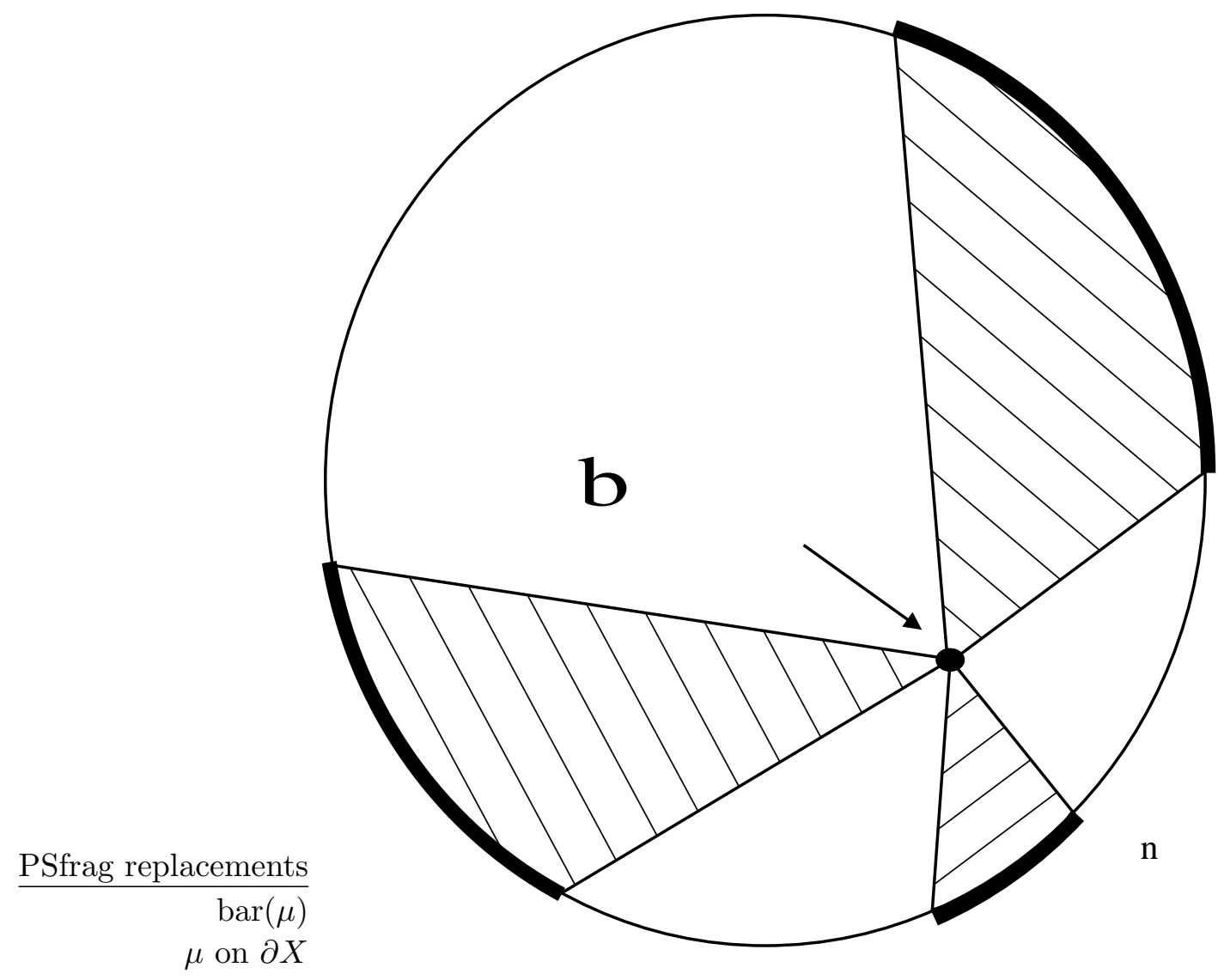

Figure 2: The barycenter of a measure.

Differentiating (5) and applying the Implicit Function Theorem then gives a formula for $\left|\operatorname{Jac} F_{s}\right|$. An application of Hölder's inequality and some further estimates then give:

$$
\left|\operatorname{Jac} F_{s}\right| \leq\left(\frac{s}{\sqrt{n}}\right)^{n} \frac{\operatorname{det}\left(\int_{\partial_{\infty} X} d B_{\left(F_{s}(y), \theta\right)}^{2}(\cdot) d \sigma_{y}^{s}(\theta)\right)^{1 / 2}}{\operatorname{det}\left(\int_{\partial_{\infty} X} D d B_{\left(F_{s}(y), \theta\right)}(\cdot, \cdot) d \sigma_{y}^{s}(\theta)\right)}
$$

Note that all of this works as long as $F_{s}$ is well-defined.

We wish to bound the right hand side of (6). In order to do this we first convert the problem to a Lie groups problem. This is where we use crucially the assumption that $M$ is locally symmetric. An easy argument allows us 
to assume that the universal cover of $M$ is irreducible, which we now do.

Recall Property (4) of Patterson-Sullivan measures, which states that in the locally symmetric case, the support of each Patterson-Sullivan is contained in a specific $\Gamma$-orbit which may also be identified with the Furstenberg boundary corresponding to the Lie group $G=\operatorname{Isom}(\widetilde{M})$ of isometries of the symmetric space $\widetilde{M}$. From this one can deduce the following:

Key fact: The expression $\partial_{\infty} X$ in (6) can be replaced by the maximal compact subgroup $K \subset G=\operatorname{Isom}(X)$.

To further simplify the right hand side of (6), we combine :

- Eigenvalue estimates on $D d B$, and

- The fact that, for $M_{i}$ positive semidefinite, $\operatorname{det}\left(\sum M_{i}\right)$ is a nondecreasing homogeneous polynomial in eigenvalues of the $M_{i}$.

to obtain

$$
|\operatorname{Jac} F| \leq C \frac{\left(\operatorname{det} \int_{K} O_{\theta}\left(\begin{array}{ll}
1 & 0 \\
0 & 0
\end{array}\right) O_{\theta}^{*} d \sigma_{y}(\theta)\right)^{\frac{1}{2}}}{\operatorname{det} \int_{K} O_{\theta}\left(\begin{array}{ll}
0 & 0 \\
0 & I
\end{array}\right) O_{\theta}^{*} d \sigma_{y}(\theta)}
$$

where $I$ is the identity matrix of $\operatorname{size} n-\operatorname{rank}(X)$, the matrix $O_{\theta}$ is an element of $K$, and the constant $C$ depends only on $n$ and on the Ricci curvatures of $M, N$. We remark that the eigenvalues of $D d B$ are determined purely by algebraic data attached to the Lie algebra of $G$.

The strategy now is to bound the right hand side of (7) over all probability measures on $K$. In some sense this is the weakness of the barycenter method: without knowing additional constraints on the measure $\sigma_{y}$, one does not always obtain a bound as needed to prove Theorem 4, much less Conjecture 1 , at least for certain locally symmetric manifolds; such examples which show that the method can fail are given in $\S 6$ below.

Some bounds on the right hand side of (7) are possible in most cases, however; the main technique is called "eigenvalue matching" in [CF2]. The idea is that for each small eigenvalue in the denominator, one tries to find two comparably small eigenvalues in the numerator. The hard part is to make "comparably small" independent of the measure. This involves a detailed analysis of action of the maximal compact subgroup $K$ on subspaces of Lie algebra of $G=\operatorname{Isom}(X)$ (a simple Lie group). For details see [CF2].

The first basic fact used here is that the kernel of the operator in the numerator of the right hand side of (7) contains the cokernel of the operator 
in the denominator. The second main ingredient is the following proposition, which gives a $K$-invariant subspace perpendicular to $K$-orbit of a flat, and of twice the dimension of the flat $(=\operatorname{rank}(X))$. This fact is precisely where the hypothesis that $G \neq \mathrm{SL}(3, \mathbf{R})$ is used.

Proposition 13 (Eigenspace matching). There is a constant $C$, depending only on $\operatorname{dim} X$ such that for any subspace $V \subset T_{x} X$ with $\operatorname{dim} V \leq$ $\operatorname{rank}(X)$ there is a subspace $V^{\prime} \subset V^{\perp}$ of dimension $2 \cdot \operatorname{dim} V$ satisfying

$$
\measuredangle\left(O_{\theta} \cdot V^{\prime}, A^{\perp}\right) \leq C \measuredangle\left(O_{\theta} \cdot V, A\right)
$$

for all $O_{\theta} \in K$.

\subsection{The perfect bound in rank one}

When $M$ has real-rank one, i.e. when $M$ is negatively curved, BessonCourtois-Gallot are able to obtain the infinitesimal rigidity described on page 6 (and hence a proof of the entropy rigidity conjecture) because of the following remarkable phenomenon. It is possible to write the operator in the denominator of the right hand side of the inequality (7) explicitly in terms of the operator in the numerator and the complex structure on the symmetric space; see [BCG2] for details. For example, for the case when $M$ is real-hyperbolic, the denominator operator is the identity minus the numerator. They are then able to solve the minimization problem explicitly by using (quite nontrivial) linear algebra.

\section{Negative results in various settings}

In this section we describe in greater detail some negative results in the settings given in the table on page 5

\subsection{Finsler metrics}

Given a manifold, $M$, if we equip each tangent space $T_{x} M$ with a norm $F_{x}$ which depends smoothly on $x$ then this gives rise to a Finsler metric by taking the distance between two points $x$ and $y$ to be the infimum of lengths of curves connecting $x$ to $y$ where length is measured using the norm on their tangent vectors. For our purposes we shall assume that the unit ball in each $T_{x} M$ given by the norm is strictly convex with $C^{2}$ boundary. This is a common assumption since it allows us to form a positive definite inner 


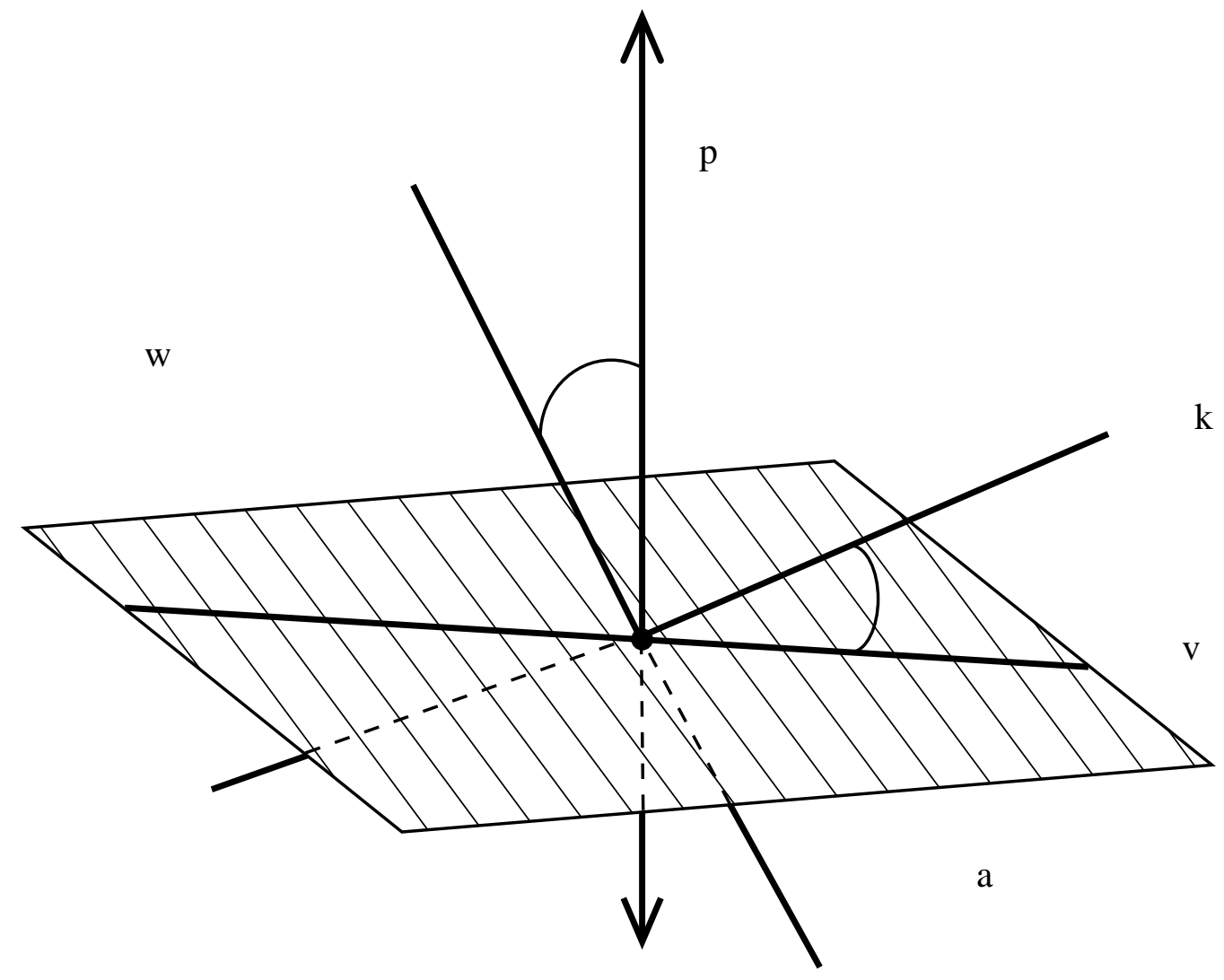

Figure 3: Eigenvalue matching.

product, $g_{u}$, for each $u \in T_{x} M$ whose matrix in local coordinates $\left(x_{i}, \dot{x}_{i}\right)$ for $T M$ is given by entries

$$
\frac{\partial^{2} F^{2}}{\partial \dot{x_{i} \partial \dot{x_{j}}}}(u)
$$

As expected, when $F$ comes from a Riemannian inner product then the $g_{u}$ all equal the inner product on $T_{x} M$. The notion of a geodesic flow makes sense for $F$ as it does for the Riemannian case, and therefore we have a dynamical notion of topological entropy.

Let $B_{x}^{F}(R)$ be the ball of radius $R$ in $T_{x} M$ in the norm $F$. For any Riemannian metric $g$ on $T M$ we can similarly define $B_{x}^{g}(R)$. Then the 
volume form for $F$ is defined at each point $x$ to be,

$$
d F(x):=\frac{\operatorname{Vol}_{g} B_{x}^{g}(1)}{\operatorname{Vol}_{g} B_{x}^{F}(1)} d g(x),
$$

which is independent of the choice of inner product $g$. In particular, the quantities $\operatorname{Vol}_{F}(M)$ and $h_{\mathrm{Vol}}(F)$ make sense.

For deformations of a single Finsler metric, we can keep $d F$ fixed by simply keeping the $\operatorname{Vol}_{g} B_{x}^{F}(1)$ fixed for each $x$ relative to a fixed inner product $g$. Keeping the topological entropy constant as well is more subtle. P. Vérovic ([Ve]) has shown for any volume-preserving deformation $F_{t}$ for $t \in(-\epsilon, \epsilon)$ of a compact hyperbolic manifold through Finsler metrics where $F_{0}$ is a hyperbolic Riemannian metric,

$$
\left.\frac{d}{d t}\right|_{t=0} \mathrm{~h}_{\mathrm{top}}\left(F_{t}\right)=0 .
$$

For surfaces he shows the same result for deformations which preserve the Liouville volume of each unit tangent sphere. Moreover, there exist oneparameter families $F_{t}$ where topological entropy can be held constant; in particular the rigidity part of Theorem 2 does not hold when the class of metrics is extended to Finsler metrics.

In higher rank things get worse: Vérovic also proved in [Ve] that for compact higher rank symmetric spaces $\left(M, g_{0}\right)$, there exists a Finsler metric $F_{0}$ (invariant under all local isometries) with $\operatorname{Vol}_{F_{0}}(M)=\operatorname{Vol}_{g_{0}}(M)$ and $h_{\mathrm{Vol}}\left(F_{0}\right)<h_{\mathrm{Vol}}\left(g_{0}\right)$; that is, the locally symmetric metric on a higher rank manifold does not minimize normalized entropy among Finsler metrics.

Any invariant Finsler metric is determined by its values on a single maximal flat $A$. In fact, if $\mathfrak{a}$ is the corresponding maximal abelian subalgebra and $\Lambda^{+}$is a set of positive roots $\alpha$ each with multiplicity $m_{\alpha}$ then $F_{0}$ is given by

$$
F_{0}(H):=\sum_{\alpha \in \Lambda_{\mathfrak{a}}^{+}} m_{\alpha}|\alpha(H)|
$$

for each $H \in \mathfrak{a}$.

In [BCG3] Besson, Courtois and Gallot asked the following intriguing question.

Question 14. Does Vérovic's Finsler metric $F_{0}$ minimize normalized entropy among all Finsler metrics? 


\subsection{Magnetic field flows}

Let $(M, g)$ be a Riemannian manifold, let $\pi: T M \longrightarrow M$ be natural projection from the tangent bundle $T M$ of $M$. The metric $g$ determines an isomorphism of $T M$ with the cotangent bundle; let $\omega_{0}$ be the symplectic 2 -form on $T M$ formed by pulling back the canonical symplectic form on $T^{*} M$ by this isomorphism. Now consider any closed 2 -form $\Omega$ on $M$. Then for any $\lambda \geq 0$ it is easy to verify that

$$
\omega_{\lambda}=\omega_{0}+\lambda \pi^{*} \Omega
$$

is also a symplectic form on $T M$.

Consider the usual Hamiltonian

$$
H_{x}(v):=\frac{1}{2} g_{x}(v, v)
$$

and define the $\lambda$-magnetic flow of the pair $(g, \Omega)$, denoted $\phi_{\lambda}$, to be the Hamiltonian flow of $H$ with respect to $\omega_{\lambda}$. Consider the bundle map $Y$ : $T M \longrightarrow T M$ given implicitly by

$$
\lambda \Omega_{x}(u, v)=g_{x}\left(Y_{x} u, v\right)
$$

Then for $v \in T M$ the curve $t \mapsto \phi_{\lambda}^{t}(v)=(\gamma(t), \dot{\gamma}(t))$ is characterized by the fact that

$$
\nabla_{\dot{\gamma}(t)} \dot{\gamma}(t)=Y_{\gamma(t)} \dot{\gamma}(t)
$$

where $\nabla$ is the connection for the metric $g$; in particular $\phi_{0}^{t}$ is just the geodesic flow for $g$.

Now we consider the case where $M$ admits an Anosov geodesic flow. For instance we could take $M$ to be any negatively curved compact manifold. In $[\mathrm{PaPa} 1]$ and $[\mathrm{PaPa} 2], \mathrm{G}$. and M. Paternain show that for $\Omega \neq 0$ the function $\mathrm{h}_{\mathrm{top}}\left(\phi_{\lambda}\right)$ is strictly decreasing for $\lambda \geq 0$ in the (nonempty) interval containing $\lambda=0$ for which $\phi_{\lambda}$ is an Anosov flow. K. Burns and G. P. Paternain further show that $\mathrm{h}_{\text {top }}\left(\phi_{\lambda}\right)$ is decreasing between "Anosov intervals" (see [BP]). In this paper the authors also give an example of a higher genus surface $M$, with a certain choice of $\Omega$, where $\mathrm{h}_{\text {top }}\left(\phi_{\lambda}\right)$ increases for $\lambda$ in between disjoint intervals where $\phi_{\lambda}$ is Anosov. Therefore, while metrics corresponding to local maxima and minima for $\mathrm{h}_{\mathrm{top}}\left(\phi_{\lambda}\right)$ may be special, no standard entropy rigidity exists for such flows. 


\subsection{Metric entropy}

Lastly, we mention the work of L. Flaminio in $[\mathrm{Fl}]$. The metric entropy is the measure-theoretic entropy of the Liouville measure on the unit tangent bundle of $M$. The measure-theoretic entropy of the Bowen-Margulis measure is the same as the topological entropy, which in turn is the same as the volume entropy. Hence for hyperbolic metrics, for instance, the metric entropy and volume entropy coincide. In general the topological entropy is always greater than or equal to the metric entropy. Flaminio shows that the metric entropy for volume preserving deformations of a hyperbolic metric on a 3-manifold $M$ does not have a local maximum at the hyperbolic metric. This is in contrast to the two dimensional case where A. Katok [Ka] showed that the metric entropy does have a local maximum there.

\section{Positive results in various settings}

In this section we describe some known cases where a locally symmetric metric structure minimizes $h(g)$ for a suitable normalization.

\subsection{Finsler metrics}

Recall the definition of a Finsler metric given above. We call a Finsler metric $F$ reversible if it satisfies $F(-v)=F(v)$ for all $v \in T M$. Moreover we define an eccentricity factor $N(F)$ for a Finsler metric $F$ on a manifold $X$ to be

$$
N(F):=\max _{x \in X} \max _{u \in S_{x}^{F}(1)} \max _{v \in S_{x}^{g_{u}}(1)} \frac{F_{x}(v)^{n} \operatorname{Vol}_{g_{u}}\left(B_{x}^{F}(1)\right)}{\operatorname{Vol}_{g_{u}}\left(B_{x}^{g_{u}}(1)\right)}
$$

where $S_{x}^{F}(1)$ and $S_{x}^{g_{u}}(1)$ are the unit spheres in the norms $F$ and $g_{u}$ respectively. For this setting we redefine the volume entropy functional ent to be

$$
\operatorname{ent}(F):=N(F) h(F)^{n} \operatorname{Vol}_{F}(X)
$$

where $n$ is the dimension of $X$.

To each direction in $T_{x} X$ we may ascribe a Riemannian metric, and consider the corresponding curvature tensor. These are the flag curvatures (see $[\mathrm{BaCS}]$ ). With this notation, J. Boland and F. Newberger [BN] proved the following theorem.

Theorem 15 (Finsler entropy rigidity). Let $\left(X, g_{0}\right)$ be a compact, $n$ dimensional, locally symmetric Riemannian manifold of negative curvature 
$(n \geq 3)$ and $(Y, F)$ a compact, reversible, Finsler manifold of negative flag curvature that is homotopy equivalent to $\left(X, g_{0}\right)$. Then

$$
1 \leq \frac{\operatorname{ent}(F)}{\operatorname{ent}\left(g_{0}\right)}
$$

with equality if and only if $F$ is homothetic to $g_{0}$

Notice that if $F$ is Riemannian then $N(F)=N\left(g_{0}\right)=1$ and so this reduces to the usual entropy rigidity theorem for the case of maps homotopic to the identity.

\subsection{Foliations}

Let $N$ and $M$ be compact topological manifolds supporting continuous foliations $\mathcal{F}_{N}$ and $\mathcal{F}_{M}$ by leaves which are smooth Riemannian manifolds, and such that the metrics on the leaves vary continuously in the transverse direction. We suppose that the leaves of $\mathcal{F}_{M}$ are locally isometric to $n$ dimensional symmetric spaces of negative curvature, $n \geq 3$; by continuity of the metrics these are all locally homothetic to a fixed symmetric space $\left(\widetilde{X}, g_{o}\right)$.

For any leaf $\left(L, g_{L}\right)$, we may define

$$
\bar{h}\left(g_{L}\right)=\limsup _{R \longrightarrow \infty} \frac{\log \operatorname{Vol}(B(x, R))}{R}
$$

where $B(x, R)$ is the ball of radius $R$ in the universal cover $\widetilde{L}$ of $L$. Similarly we can define $\underline{h}\left(g_{L}\right)$ using the $\liminf { }_{R \longrightarrow \infty}$. These numbers do not depend on the choice of $x$. We then define the volume growth entropy $h\left(g_{L}\right)$ to be

$$
h\left(g_{L}\right)=\inf \left\{s>0 \mid \int_{0}^{\infty} e^{-s t} \operatorname{Vol} S(x, t) d t<\infty\right\}
$$

where $S(x, t)$ is the sphere of radius $t$ about $x$ in the universal cover $\widetilde{L}$ of $L$. This quantity is independent of $x \in L$. Hence we can define a function $f: N \longrightarrow[0, \infty]$ by letting $f(x)$ be $h\left(g_{L}\right)$ for the leaf $L$ containing $x$. Of course $f$ is constant on each leaf.

The function $f$ is also measurable. This follows from the fact that the transverse continuity of the leafwise metrics implies that for each $R$, the function

$$
x \mapsto \int_{0}^{R} e^{-s t} \operatorname{Vol} S(x, t) d t
$$


is continuous on $N$. On $\left(M, \mathcal{F}_{M}\right)$ the entropy is constant and we denote it by $h\left(g_{o}\right)$. From the definition of $h\left(g_{L}\right)$ it follows almost immediately that

$$
\underline{h}\left(g_{L}\right) \leq h\left(g_{L}\right) \leq \bar{h}\left(g_{L}\right)
$$

For the foliation $\left(N, \mathcal{F}_{N}\right)$ we assume that the leaves $\left(L, g_{L}\right)$ are strictly negatively curved, and satisfy the stronger condition that for all $x, y \in L$ there is a $\delta<h\left(g_{L}\right)$ such that

$$
\limsup _{R \longrightarrow \infty} \frac{\operatorname{Vol} S(x, R)}{\operatorname{Vol} S(y, R)} \leq C e^{\delta d(x, y)}
$$

where $S(x, R)$ is the sphere of radius $R$ in $L$. Such a leaf will be called a Patterson-Sullivan manifold.

Finally, assume we have a leaf-preserving homeomorphism

$$
f:\left(N, \mathcal{F}_{N}\right) \rightarrow\left(M, \mathcal{F}_{M}\right)
$$

which is leafwise $C^{1}$ with transversally continuous leafwise derivatives, but not necessarily transversally differentiable. Equip the foliation $\left(N, \mathcal{F}_{N}\right)$ with any choice of finite, transverse, holonomy quasi-invariant measure $\nu$ (see Hurder [Hu] or Zimmer [Zi] for the definition and existence). Holonomy quasi-invariance simply means that the push forward of $\nu$ under any holonomy map is in the same measure class as $\nu$. This measure $\nu$ provides us with a global finite measure $\mu_{N}$ on $N$ which is locally a product of $\nu$ with the Riemannian volumes $\mathrm{dvol}_{L}$ of the leaves $L$. In this setting J. Boland and the first author $([\mathrm{BC}])$ showed the following.

Theorem 16 (Foliated entropy rigidity, I). Let $\left(N, \mathcal{F}_{N}\right)$ be a continuous foliation of the compact manifold $N$ such that $\nu$-almost every leaf is a Patterson-Sullivan manifold. Suppose that $f:\left(N, \mathcal{F}_{N}\right) \longrightarrow\left(M, \mathcal{F}_{M}\right)$ is a foliation-preserving homeomorphism, leafwise $C^{1}$ with transversally continuous leafwise derivatives, and that $f_{*} \nu$-almost every leaf of $\left(M, \mathcal{F}_{M}\right)$ is a rank one locally symmetric space. Then there exists a finite measure $\mu_{M}$ on $M$ which is locally the product of $\mathrm{dvol}_{o}$ with a transverse quasi-invariant measure $\nu_{o}$ such that

$$
\int_{M} h\left(g_{o}\right)^{n} d \mu_{M} \leq \int_{N} h\left(g_{L}\right)^{n} d \mu_{N}
$$

and equality holds if and only if $\nu$-almost every leaf $\left(L, g_{L}\right)$ is homothetic to its image $\left(f(L), g_{o}\right)$. 
When the foliation admits a holonomy-invariant measure $\nu$ then we may take $\nu_{o}=f_{*} \nu$. When $\nu$ is just holonomy quasi-invariant however, then $\nu_{o}$ is the push forward of $\nu$ under the natural map $F$ defined below.

When the foliation $\left(N, \mathcal{F}_{N}\right)$ is ergodic with respect to $\nu$, then the entropy function $h\left(g_{L}\right)=h(g)$ is constant on $N$, and we get the following (see [BC]).

Corollary 17 (Foliated entropy rigidity, II). Under the same assumptions as in the main theorem, if $\left(N, \mathcal{F}_{N}\right)$ is ergodic, then $h\left(g_{o}\right)^{n} \operatorname{Vol}\left(M, \mu_{M}\right) \leq$ $h(g)^{n} \operatorname{Vol}\left(N, \mu_{N}\right)$ with equality if and only if $\nu$-almost every leaf $\left(L, g_{L}\right)$ is homothetic to $\left(f(L), g_{o}\right)$.

Remark. If $\left(N, \mathcal{F}_{N}\right)$ and $\left(M, \mathcal{F}_{M}\right)$ are foliations such that almost every leaf is compact or simply connected, then the requirement that the homeomorphism $f$ be leafwise $C^{1}$ can be dropped. In particular if the foliations have just one leaf and $\operatorname{dim} N \neq 3,4$, any homotopy equivalence induces a homeomorphism between $N$ and $M$ (see [FJ]). Therefore, when $\operatorname{dim} N \neq 3,4$, Corollary 17 recovers Theorem 2.

Of course one can also ask for foliated versions of Conjecture 1 as well.

\subsection{Finite volume manifolds}

For the case of finite volume manifolds J. Boland, J. Souto and the second author showed the following analogue of the Real Schwarz Lemma.

Theorem 18 (Volume Theorem [BCS]). Let $(M, g)$ and $\left(M_{o}, g_{o}\right)$ be two oriented complete finite volume Riemannian manifolds of the same dimension $n \geq 3$ and suppose that

$$
\operatorname{Ric}_{g} \geq-(n-1) g, \quad \text { and } \quad-a \leq K_{g_{o}} \leq-1 .
$$

Then for all proper continuous maps $f: M \longrightarrow M_{o}$,

$$
\operatorname{Vol}(M, g) \geq|\operatorname{deg}(f)| \operatorname{Vol}\left(M_{o}, g_{o}\right)
$$

and equality holds if and only if $M$ and $M_{o}$ are hyperbolic and $f$ is proper homotopic to a Riemannian covering.

When $M$ and $M_{o}$ are compact, Theorem 18 follows from a real Schwarz lemma proved by Besson, Courtois, and Gallot in [BCG5]. As in the case of all noncompact situations, to apply the method of [BCG1] and [BCG5], the fundamental difficulty is proving the properness of the natural map.

Theorem 18 implies the following. 
Corollary 19. Under the hypotheses of Theorem 18,

$$
\operatorname{Minvol}(M) \geq \operatorname{deg}(f) \operatorname{Vol}\left(M_{o}\right) .
$$

Now restrict $f$ to be degree 1 , and consider what happens when $\operatorname{Minvol}(M)=$ $\operatorname{Vol}\left(M_{o}\right)$. Bessières proved in [Be1] that if there is a degree 1 map $f: M \longrightarrow$ $M_{o}$ from the compact $n$-dimensional $M$ to the compact $n$-dimensional real hyperbolic manifold $M_{o}$ such that $\operatorname{Minvol}(M)=\operatorname{Vol}\left(M_{o}\right)$, then $M$ and $M_{o}$ are diffeomorphic. He also gave examples in [Be2] of a finite volume manifold $M$ with the same simplicial volume as a hyperbolic manifold $M_{o}$ such that there is a degree 1 map from $M$ to $M_{o}$ and $\operatorname{Minvol}(M) \leq \operatorname{Vol}\left(M_{o}\right)$ but $M$ and $M_{o}$ are not even homeomorphic. By the above corollary, $\operatorname{Minvol}(M)=$ $\operatorname{Vol}\left(M_{o}\right)$. Despite such examples, our next result ([BCS]) shows that the pointed Lipschitz limit of some subsequence of any sequence of metrics whose volumes achieve $\operatorname{Minvol}(M)$ is isometric to $M_{o}$.

Theorem 20 (Hyperbolic Minvol rigidity, finite volume case). Let $M$ and $M_{o}$ be finite volume manifolds of the same dimension $n \geq 3, M_{o}$ real hyperbolic, and $f: M \longrightarrow M_{o}$ a continuous, proper, degree 1 map. If $\operatorname{Minvol}(M)=\operatorname{Vol}\left(M_{o}\right)$, then for any sequence of metrics $g_{i}$ realizing the minimal volume of $M$, there are $p_{i} \in M$ and a subsequence $g_{i_{j}}$ such that $\left(M, p_{i_{j}}, g_{i_{j}}\right)$ converges in the pointed Lipschitz topology to a Riemannian manifold isometric to $M_{o}$.

In particular, the topology of the limit manifold changes to that of $M_{o}$.

The last main result of [BCS] is the finite volume version of the entropy rigidity result found in [BCG1].

Theorem 21 (Rank one entropy rigidity, finite volume case). Let $(M, g)$ be an $n$-dimensional finite volume manifold of nonpositive sectional curvature, $n \geq 3$, and $h(g)$ its volume growth entropy. Let $\left(M_{o}, g_{o}\right)$ be an $n$-dimensional finite volume rank one locally symmetric manifold and $h\left(g_{o}\right)$ its volume growth entropy. If $f: M \longrightarrow M_{o}$ is a continuous, proper map of degree $\operatorname{deg}(f)>0$, then

$$
h(g)^{n} \operatorname{Vol}(M, g) \geq \operatorname{deg}(f) h\left(g_{o}\right)^{n} \operatorname{Vol}\left(M_{o}, g_{o}\right)
$$

and equality holds if and only if $f$ is proper homotopic to a Riemannian covering.

As in [BCG2], this gives a quick proof of the Mostow rigidity theorem for finite volume negatively curved locally symmetric manifolds. 


\subsection{Quasifuchsian representations}

The barycenter method has recently been applied by Besson-Courtois-Gallot [BCG5] to convex cocompact (infinite covolume) representations, generalizing Bowen's rigidity theorem about quasifuchsian groups. We now describe one of the several results in [BCG5] along these lines.

Let $X$ be a negatively curved manifold. A faithful representation $\rho$ : $\Gamma \longrightarrow \operatorname{Isom}(X)$ is convex cocompact if $\rho(\Gamma)$ acts cocompactly on the convex hull of its limit set $\Lambda(\rho(\Gamma))$. Attached to each $\rho$ is a number, namely the Hausdorff dimension $\operatorname{dim}_{\mathcal{H}}(\rho(\Lambda(\Gamma)))$. Amazingly, this single number can be used to characterize the totally geodesic representations, i.e. those representations leaving invariant a totally geodesic submanifold. The following theorem was originally proved by Pansu, Bourdon, and Yue, generalizing an earlier theorem of Bowen. Besson-Courtois-Gallot gave another proof in [BCG5] using the barycenter method.

Theorem 22 (Quasifuchsian rigidity). Let $\Gamma$ be the fundamental group of a closed, hyperbolic $n$-manifold, and let $X$ be a connected, simply-connected manifold with sectional curvature $\leq-1$. Then

$$
\operatorname{dim}_{\mathcal{H}}(\rho(\Lambda(\Gamma))) \geq n-1
$$

with equality if and only if $\rho$ is totally geodesic.

There is also a version for complex hyperbolic manifolds (see [BCG5]). The idea of the proof is to define the barycenter map as we discussed above, and to bound the Jacobian of this map; Theorem 22 then follows reasonably quickly. The main difficulty is that the dimensions of the domain and target spaces are different. To repair this one notes that $\rho$ gives a quasi-isometric embedding between negatively curved spaces, hence a homeomorphic embedding of one boundary at infinity into another. The general technique can then be carried out once one finds the right notion of volume.

\subsection{Alexandroff Spaces}

In this section we report on recent work of P. Storm, who extended some of the results of [BCG1] to certain Alexandroff space domains within the same bilipschitz class as a fixed hyperbolic manifold. All of the results can be found in [St].

In brief, an Alexandroff space $X$ with curvature bounded below by -1 is any complete, locally compact metric space of finite Hausdorff dimension, such that every point $x \in X$ has a neighborhood containing a geodesic 
triangle such that the comparison triangle in $\mathbf{H}^{2}$ with the same side lengths has the property that the distance form any vertex of the triangle to any point on the opposite side is shorter than the corresponding distance on the triangle in $X$. Storm's main result in [St] is the following.

Theorem 23 (Alexandroff domain). Let $(X, d)$ be an Alexandroff space with curvature bounded below by -1 , and $Y$ a closed, hyperbolic n-manifold, $n \geq 3$. If there is a bilipschitz homeomorphism between $X$ and $Y$, then

$$
\operatorname{Vol}(X) \geq \operatorname{Vol}(Y)
$$

The proof of this theorem is at first glance unrelated to the method of Besson, Courtois, and Gallot as outlined in the opening sections of this paper. Nevertheless, it is actually of the same vein. The main technique is to extend the idea of Spherical Volume as presented in [BCG1] to this new setting. Instead of going through the (perhaps insurmountable) difficulty of forming a barycenter map from $X$ to $Y$, one can in a sense stop "half-way" and attempt to do the analysis in $\mathcal{M}(X)$ and $\mathcal{M}(Y)$. Since the embedding via measures is in essence an $L^{1}$ approach it is preferable to modify this to an $L^{2}$ approach which leaves us working in a Hilbert space.

More precisely, one embeds $\widetilde{X}$ into $L^{2}(\widetilde{X})$ in a $\pi_{1}$-equivariant way via the map

$$
\Phi_{s}(x):=e^{s / 2 d(x, \cdot)}
$$

for any $s>h(X)$, and then projects this to the unit Hilbert sphere $S^{\infty}(\widetilde{X})$. We recall that this is the embedding achieved by using the square root of the Radon-Nikodym derivatives of the un-normalized $\sigma_{y}^{s}$, which are in $L^{2}$ by definition of the volume growth entropy $h(X)$. If $f: \tilde{Y} \longrightarrow \widetilde{X}$ represents a bilipschitz $\pi_{1}$-equivariant map given by the hypotheses of the theorem, then the map

$$
\widetilde{I}: L_{2}(\widetilde{X}) \longrightarrow L_{2}(\widetilde{Y})
$$

given by

$$
\mathcal{I}(g):=(g \circ f) \operatorname{Jac}(f)
$$

is a $\pi_{1}$-equivariant isometry. Hence it restricts to $\mathcal{I}: S^{\infty} \widetilde{X} \longrightarrow S^{\infty} \widetilde{Y}$. The composition

$$
\widetilde{F}:=\mathcal{I} \circ \Phi_{s} \circ f: \tilde{Y} \longrightarrow S^{\infty} \tilde{Y}
$$

descends to a map $F: Y \longrightarrow S^{\infty}(Y)$ with image in the positive orthant.

For such maps there is a notion of volume, and without defining it, we simply point out that spherical volume is the infimum of the volume of such 
maps. In particular

$$
\operatorname{Vol}(F) \geq \operatorname{Spherical} \operatorname{Vol}(Y)=\left(\frac{(n-1)^{2}}{4 n}\right)^{n / 2} \operatorname{Vol}(Y)
$$

The last equality was proven by Besson-Courtois-Gallot in [BCG1].

Since Alexandroff spaces are in a sense Riemannian manifolds off of a measure zero set, and $Y$ is a Riemannian manifold, Storm can use standard integration theory. Of course we now come to the difficult (and omitted) part of the proof of Theorem 23, which is to show

$$
\operatorname{Vol}(F) \leq\left(\frac{s^{2}}{4 n}\right)^{n / 2} \operatorname{Vol}(X)
$$

It then follows that

$$
\operatorname{Vol}(X) \geq\left(\frac{n-1}{s}\right)^{n} \operatorname{Vol}(Y)
$$

The final step is to apply a result of Burago-Gromov-Perel'man which states that for Alexandroff spaces with curvature bounded below by $-1, h(X) \leq$ $n-1$; Theorem 23 then follows.

The main applications of Theorem 23 are to two important classes of Alexandroff spaces which arise naturally in the study of hyperbolic manifolds: doublings of convex cores and cone manifolds.

\subsubsection{Convex Cores}

The (metric) double $D C_{M}$ of the convex core $C_{M}$ of a convex cocompact manifold $M$ is the result of identifying the boundaries of two copies of $C_{M}$ and then extending the induced metric on each copy of $C_{M}$ to the whole. While as a topological manifold $D C_{M}$ can always be smoothed (e.g. by taking the double a neighborhood of $C_{M}$ ), the point is that any Riemannian metric on the resulting smooth manifold cannot agree with the metric on each copy of $C_{M}$ as a subspace, unless these had totally geodesic boundaries to begin with. On the other hand, $D C_{M}$ naturally carries the structure of an Alexandroff space with Alexandroff curvature bounded below by -1 and Theorem 23 applies.

Let $\mathrm{CC}(N)$ be the space of complete convex cocompact hyperbolic manifolds diffeomorphic to the interior of smooth compact $n$-manifold $N$. In analogy with the volume entropy results discussed in this paper, one wants to know that the topological invariant

$$
\mathcal{V}(N):=\inf _{M \in \mathrm{CC}(N)}\left\{\text { volume of the convex core } C_{M} \text { of } M\right\}
$$


is minimized for certain special hyperbolic manifolds.

It is consequence of Thurston's work together with Mostow Rigidity that a 3-manifold $N$ is acylindrical if and only if there exists unique $M_{0} \in \mathrm{CC}(N)$ such that $\partial C_{M_{0}}$ is totally geodesic (see [Th2]). Combining this with Theorem 23 , Storm obtains the following.

Corollary 24. . Let $N$ be an acylindrical compact irreducible 3-manifold such that $C C(N)$ is nonempty. Then there exists a unique $M_{0} \in C C(N)$ such that $\mathcal{V}(N)=\operatorname{Vol}\left(C_{M_{0}}\right)$. Moreover, $\partial C_{M_{0}}$ is totally geodesic.

In fact he shows that any $C_{M_{0}}$ with totally geodesic boundary is the unique minimizer of $\operatorname{Vol}\left(C_{N}\right)$ among hyperbolic $N$ homotopy equivalent to $M_{0}$. (Note we are using that $M_{0}$ is tame). We should also mention that Bonahon had previously shown in [Bon] that $M_{0}$ is a strict local minimum of $\operatorname{Vol}\left(C_{N}\right)$.

Remark. Storm is able to also obtain results giving exact relations between the Gromov norm of such $D C_{N}$ and covers in terms of $\mathcal{V}(N)$.

\subsubsection{Application to Cone Manifolds}

Theorem 23 may also be applied to cone-manifolds with all cone angles $\leq 2 \pi$.

Definition ([CHK], page 53). An $n$-dimensional cone-manifold is a topological manifold, $M$, which admits a triangulation giving it the structure of a PL manifold and $M$ is equipped with a complete path metric such that the restriction of the metric to each simplex is isometric to a geodesic simplex of constant curvature $K$. The singular locus $\Sigma$ consists of the points with no neighborhood isometric to a ball in a Riemannian manifold.

It follows that

- Sigma is a union of totally geodesic closed simplices of dimension $n-2$.

- At each point of $\Sigma$ in an open $(n-2)$-simplex, there is a cone angle which is the sum of dihedral angles of $n$-simplices containing the point.

Notice that cone-manifolds whose singular locus has vertices are allowed.

Lemma 25 ([BGP], page 7$)$. If all cone angles of $n$-dimensional conemanifold $M$ are $\leq 2 \pi$, and $K \geq-1$, then $M$ is an Alexandroff space with curvature bounded below by -1 . 
An $n$-dimensional cone-manifold clearly has Hausdorff dimension $n$. Therefore Theorem 23 implies the following.

Corollary 26. Let $M$ be an 3-dimensional cone-manifold with all cone angles $\leq 2 \pi$ and $K \geq-1$. Let $M_{0}$ be a closed hyperbolic 3-manifold. If $M$ and $M_{0}$ are homeomorphic then

$$
\operatorname{Vol}(M) \geq \operatorname{Vol}\left(M_{0}\right)
$$

All of these corollaries have only slightly weaker generalizations to any dimension greater than 2 . We refer the reader to [St]. There are other results whose methods follow along these lines; in particular we would like to point out the work relating to Einstein metrics, the Gromov norm, and simplicial volume for manifolds and covers carried out by A. Sambusetti (e.g. [Sam1, Sam2]). However, these recent results have already been well surveyed in [BCG5].

\section{Cautionary examples}

Our proof in [CF2] of the Degree Theorem (Theorem 4) can be viewed as a step towards the Entropy Rigidity Conjecture (Conjecture 1), or at least the inequality of that conjecture; to do this one "only" needs to prove the inequality (3) on page 7 with the lowest possible $C$, namely $C=\left(\frac{h(g)}{h\left(g_{0}\right)}\right)^{n}$.

While the value of $C$ which comes out of the proof (see [CF2]) of inequality (3) can be explicitly computed, finding the best $C$ seems much harder. In fact, it soon became clear to us that the barycenter method applied without a priori constraints on the types of measures considered is not sufficiently precise to obtain the rigidity aspect of the theory, or even to prove that the locally symmetric metric minimizes entropy. In this section we describe some explicit examples which demonstrate some of the problems.

The Jacobian estimates on the Besson-Courtois-Gallot map $F$ are obtained by bounding the right hand side of (7), that is, bounding

$$
\frac{\left(\operatorname{det} \int_{K} O_{\theta}\left(\begin{array}{ll}
1 & 0 \\
0 & 0
\end{array}\right) O_{\theta}^{*} d \sigma_{y}(\theta)\right)^{\frac{1}{2}}}{\operatorname{det} \int_{K} O_{\theta}\left(\begin{array}{ll}
0 & 0 \\
0 & I
\end{array}\right) O_{\theta}^{*} d \sigma_{y}(\theta)}
$$

independently of the measure. In other words, no special property of the measure $d \sigma_{y}$ is used. This is also the case in the proof of Entropy Rigidity in rank one (see [BCG2]). 
We recall that in a symmetric space of noncompact type, the Furstenberg boundary is the space of Hausdorff equivalence classes of (maximal) Weyl chambers; this space can be identified with $G / P$, where $P$ is a minimal parabolic subgroup of $G$. This in turn is naturally homeomorphic to $K / M$ where $K$ is the maximal compact subgroup of $G$ and $M$ is the centralizer of $\mathfrak{a}$ in $K$. Since $K$ is isomorphic to $K_{p}$, isotropy subgroup of $p \in X$, and $M$ is isomorphic to $M_{p}$, the centralizer of a Weyl chamber in $\mathcal{F}_{p}$, we may interpret the Furstenberg boundary geometrically. Namely it is naturally identified to any $G$-orbit of a regular point in $\partial X$. We will denote by $\partial_{F} X$ the particular orbit of the point at infinity corresponding to the normalized barycenter $b^{+}$of positive root vectors. By the above, $\partial_{F} X$ projects to the unit tangent sphere $S_{p} X$ as the orbit of $b^{+}$by $K_{p}$.

Hence if we write $J(\mu)$ for the square of the right hand side of the expression (6) with $\sigma_{y}^{s}$ replaced by $\mu$, then we would like for it to be uniquely maximized when $\mu$ is the projection of Haar measure on $K$. Here we only care about maximizing over the space of $M_{p}$-invariant $\mu$ supported on $K_{p}$.

\subsection{An example in $\mathrm{H}^{2} \times \mathrm{H}^{2}$}

In the case of $X=\mathbf{H}^{2} \times \mathbf{H}^{2}$ the Furstenberg boundary $\partial_{F} X$ is simply the torus $S^{1} \times S^{1}$. We will parameterize this by $\left(e^{i t_{1}}, e^{i t_{2}}\right)$. Let $M$ be a compact quotient of $X$ and let $f: M \longrightarrow M$ a continuous map. We first consider the case $f=\mathrm{Id}$ and the simple two-parameter family of probability measures $\mu_{a, b}$ for $a, b>0$ on $\partial_{F} X$ given by

$$
d \mu_{a, b}=\frac{2+b+2 a \cos \left(\frac{t_{1}}{2}\right)^{4}+b \cos \left(t_{2}\right)}{(8+3 a+4 b) \pi^{2}} d \mu_{0}
$$

where $\mu_{0}$ the unit Haar measure on the torus. Note that $\mu_{0,0}$ is Haar measure. For this family, by integrating over the torus we can compute the expression for

$$
J_{0}\left(\mu_{a, b}\right):=J\left(\mu_{a, b}\right) / J(\text { Haar })
$$

to be

$$
J_{0}\left(\mu_{a, b}\right)=\frac{4\left(5 a^{2}+24 a(2+b)+16(2+b)^{2}\right)^{2}}{(8+3 a+4 b)^{2}(5 a+8(2+b))(7 a+8(2+b))} .
$$

Note that the measures $\mu_{0, b}$ are distinct, but that $J\left(\mu_{0, b}\right) / J\left(\mu_{0,0}\right)=1$ identically. In particular the Haar measure does not uniquely minimize this quantity. This may not be that surprising in light of the fact that $\mathbf{H}^{2}$ does not have a unique minimizer either, nor is there a entropy rigidity theorem for $X$, at least not for reducible lattices. 
PSfrag replacements

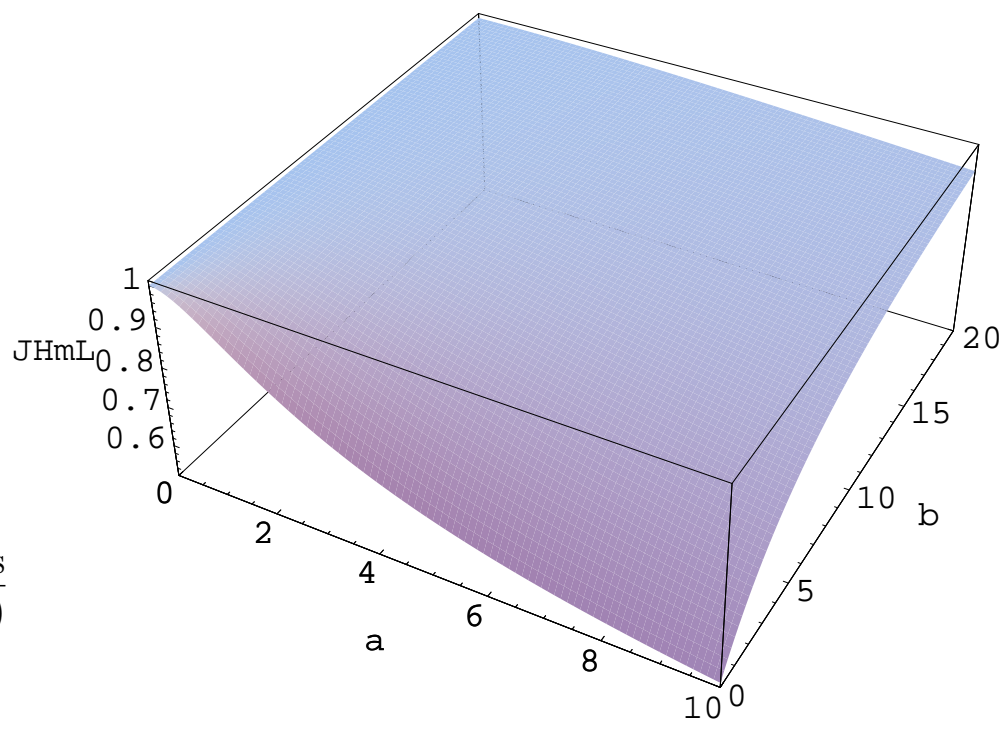

Figure 4: Graph of $J\left(\mu_{a, b}\right) / J($ Haar $)$ for several values of $a$ and $b$.

\subsection{An example in $\mathrm{SL}_{3}(\mathbf{R}) / \mathrm{SO}_{3}(\mathbf{R})$}

Now we examine the case of $X=\mathrm{SL}(3, \mathbf{R}) / \mathrm{SO}(3)$. Suppose for a single flat $\mathcal{F}_{x}$ and a sequence of $y_{i} \in \mathcal{F}_{x}$, the measures $\sigma_{y_{i}}^{s}$ tend to the sum of Dirac measures

$$
\mu=\frac{1}{6} \sum_{i=1^{6}} \delta_{b_{i}^{+}(\infty)}
$$

where the $b_{i}^{+}$are all the images of $b^{+}$under the Weyl group in $\mathcal{F}_{x}$. Hence there is one atom for each Weyl chamber at $x$ and they are symmetrically placed making $x$ the barycenter of $\mu$. Then we claim that if $N=M$ and $\widetilde{f}$ induces the identity map on $\partial \mathcal{F}_{x}$ then $\operatorname{Jac}_{F_{s}}\left(y_{i}\right) \longrightarrow \infty$.

The hypotheses that $N=M$ and that $\widetilde{f}$ is identity on $\partial \mathcal{F}_{x}$, which contains the support of $\mu$, implies that $\operatorname{Jac} F_{s}\left(y_{i}\right)$ is identical in the limit $i \longrightarrow \infty$ to the estimate on the right-hand side of (6) with $\sigma_{y}^{s}$ replaced by $\mu$. Therefore it remains to show that this right-hand side is unbounded. 
First note that the sum

$$
\lim _{i \longrightarrow \infty} \sum_{j=1}^{6} d B_{\left(F_{s}\left(y_{i}\right), b_{j}^{+}(\infty)\right)}^{2}
$$

has only a 3-dimensional kernel, while

$$
\lim _{i \longrightarrow \infty} \sum_{j=1}^{6} D d B_{\left(F_{s}\left(y_{i}\right), b_{j}^{+}(\infty)\right)}
$$

has a 2-dimensional kernel. Furthermore the numerator and denominator

$$
Q_{1}:=\int_{\partial_{F} X} d B_{\left(F_{s}\left(y_{i}\right), \theta\right)}^{2} d \sigma_{y_{i}}^{s} \text { and } Q_{2}:=\int_{\partial_{F} X} D d B_{\left(F_{s}\left(y_{i}\right), \theta\right)} d \sigma_{y_{i}}^{s}
$$

degenerate homogeneously. In particular, the quantity $\operatorname{det}\left(Q_{1}\right) / \operatorname{det}\left(Q_{2}\right)^{2}$ is unbounded. This can be easily verified explicitly in the case of a sum of eight Dirac measures for which both integrals are nonsingular degenerating to the sum of the six Dirac measures given above.

\subsection{An example in $\mathrm{SL}_{4}(\mathbf{R}) / \mathrm{SO}_{4}(\mathbf{R})$}

In the case when $X=\mathrm{SL}(4, \mathbf{R}) / \mathrm{SO}(4)$, Theorem 4 holds and the quantity (6) is bounded independently of $\sigma_{y}^{s}$. Nevertheless, here we present a smooth (with respect to Haar) probability measure $\mu$ on $\mathrm{SO}(4) / M$ such that $J_{0}(\mu)$ is strictly larger than 1 . In fact, $\mu$ will be very close to the Haar measure $\mu_{0}$ only differing by the addition of a few very small and sharp "thorns" and removal of a few "drillings." What we mean by this will become evident from the final construction.

Our procedure is to consider second derivatives of the Jacobian

$$
f(t):=J_{0}\left(\frac{\mu_{0}+t \mu}{\left\|\mu_{0}+t \mu\right\|}\right)
$$

with respect to a parameter $t$ where $\mu$ is an arbitrary signed measure. In fact, if we can find such a $\mu$ for which the second derivative at zero is positive then we will show how one can obtain such an example where $\mu$ is a smooth (with respect to $\mu_{0}$ ) positive probability measure.

One can verify directly that, for general $X$,

$$
J\left(\mu_{0}\right)=\left(\frac{s^{2} / n}{(\operatorname{Tr}(D d B) / n)^{2}}\right)^{n}
$$


which for $\operatorname{SL}(4, \mathbf{R})$ works out to be

$$
\left(\frac{9 s^{2}}{40}\right)^{9}
$$

Hence, after clearing the denominator of the measures, we can rewrite

$$
f(t)=\left(\frac{40}{9}\left\|\mu_{0}+t \mu\right\|\right)^{9} \frac{\operatorname{det}\left(\int_{\partial_{F} X} d B_{\left(F_{s}(y), \theta\right)}^{2} d\left[\mu_{0}+t \mu\right](\theta)\right)}{\operatorname{det}\left(\int_{\partial_{F} X} D d B_{\left(F_{s}(y), \theta\right)}(\cdot, \cdot) d\left[\mu_{0}+t \mu\right](\theta)\right)^{2}}
$$

Taking the derivative of the log we have

$$
\partial_{t} f(t)=f(t) \partial_{t} \log f(t)
$$

Hence the second derivative is

$$
\partial_{t} \partial_{t} f(t)=f(t)\left[\left(\partial_{t} \log f(t)\right)^{2}+\partial_{t} \partial_{t} \log f(t)\right]
$$

For an invertible matrix $M$ we have the operator identities

$$
\log \operatorname{det} M=\operatorname{Tr} \log M \text { and } \partial_{t} \operatorname{Tr} \log M=\operatorname{Tr}\left(\left(\partial_{t} M\right) M^{-1}\right)
$$

Applying these identities we directly obtain

$$
\begin{array}{r}
\partial_{t} \log f(t)=\operatorname{Tr}\left(\int_{\partial_{F} X} d B_{\left(F_{s}(y), \theta\right)}^{2} d \mu(\theta)\right)\left(\int_{\partial_{F} X} d B_{\left(F_{s}(y), \theta\right)}^{2} d\left[\mu_{0}+t \mu\right](\theta)\right)^{-1}+ \\
9 \frac{\|\mu\|}{\left\|\mu_{0}+t \mu\right\|}-2 \operatorname{Tr}\left(\int_{\partial_{F} X} D d B_{\left(F_{s}(y), \theta\right)} d \mu(\theta)\right)\left(\int_{\partial_{F} X} D d B_{\left(F_{s}(y), \theta\right)} d\left[\mu_{0}+t \mu\right](\theta)\right)^{-1}
\end{array}
$$

Similarly,

$$
\begin{array}{r}
\partial_{t} \partial_{t} \log f(t)=-\operatorname{Tr}\left(\left(\int_{\partial_{F} X} d B_{\left(F_{s}(y), \theta\right)}^{2} d \mu(\theta)\right)\left(\int_{\partial_{F} X} d B_{\left(F_{s}(y), \theta\right)}^{2} d\left[\mu_{0}+t \mu\right](\theta)\right)^{-1}\right)^{2}- \\
9\left(\frac{\|\mu\|}{\left\|\mu_{0}+t \mu\right\|}\right)^{2}+2 \operatorname{Tr}\left(\left(\int_{\partial_{F} X} D d B_{\left(F_{s}(y), \theta\right)} d \mu(\theta)\right)\left(\int_{\partial_{F} X} D d B_{\left(F_{s}(y), \theta\right)} d\left[\mu_{0}+t \mu\right](\theta)\right)^{-1}\right)^{2}
\end{array}
$$

Since $f(0)=1$ and

$$
\int_{\partial_{F} X} d B_{\left(F_{s}(y), \theta\right)}^{2} d \mu_{0}(\theta)=\frac{1}{9} \mathrm{Id} \quad \text { and } \quad \int_{\partial_{F} X} D d B_{\left(F_{s}(y), \theta\right)} d \mu_{0}(\theta)=\frac{\sqrt{20}}{9} \mathrm{Id}
$$


we see that $\left.\partial_{t}\right|_{t=0} f(t)=0$ and

$$
\begin{array}{r}
\left.\partial_{t} \partial_{t}\right|_{t=0} f(t)=-9^{2} \operatorname{Tr}\left(\int_{\partial_{F} X} d B_{\left(F_{s}(y), \theta\right)}^{2} d \mu(\theta)\right)^{2}-9\left(\int_{\partial_{F} X} d \mu\right)^{2}+ \\
\frac{9^{2}}{10} \operatorname{Tr}\left(\int_{\partial_{F} X} D d B_{\left(F_{s}(y), \theta\right)} d \mu(\theta)\right)^{2} .
\end{array}
$$

Now we consider the Cartan splitting $\mathrm{SL}(4, \mathbf{R}) / K=K P$ where $P$ are the positive definite symmetric matrices with determinant 1 and we identify $K$ with the stabilizer of $F_{s}(y) . P$ is naturally identified with the 9-dimensional subspace $\mathfrak{p}$ of traceless symmetric matrices in $\mathfrak{s l}(4, \mathbf{R})$. We also have the natural representation of $K$ in $\mathrm{SO}(\mathfrak{p})$ induced by the action of $K$ on $P$ by conjugation.

By lifting $\partial_{F} X$ to $K$ we may treat the integration over $K$ and it is convenient to parameterize $K$ by $\mathbf{R}^{6} \equiv \mathfrak{s}$ the subalgebra of skew-symmetric matrices in $\mathfrak{s l}(4, \mathbf{R})$. We can choose a domain of integration $D \subset \mathbf{R}^{6}$, and integrate over this space. For $\theta \in D$ let $\mathrm{O}(\theta) \in \mathrm{SO}(\mathfrak{p})$ be the corresponding element. If $A$ is the constant diagonal matrix with diagonal $(1,0,0,0,0,0,0,0,0)$ and $B$ is the diagonal matrix with diagonal

$$
\left(0,0,0, \sqrt{\frac{2}{5}}, \sqrt{\frac{2}{5}}, \sqrt{\frac{2}{5}}, 2 \sqrt{\frac{2}{5}}, 2 \sqrt{\frac{2}{5}}, 3 \sqrt{\frac{2}{5}}\right)
$$

then relative to the appropriate choice of coordinates on $T_{F_{s}(y)} X$ we may write

$$
d B_{\left(F_{s}(y), \theta\right)}^{2}=O(\theta) A O(\theta)^{*}
$$

and

$$
D d B_{\left(F_{s}(y), \theta\right)}=O(\theta) B O(\theta)^{*}
$$

Note that the metric we are using is $\frac{1}{\sqrt{2}}$ times the one most commonly used by representation theorists.

With these notations, the expression above for $\left.\partial_{t} \partial_{t}\right|_{t=0} f(t)$ becomes

$$
\int_{D} \int_{D} q(\sigma, \tau) d \mu(\sigma) d \mu(\tau)
$$

where

$q(\sigma, \tau)=-9^{2} \operatorname{Tr}\left(O(\sigma) A O(\sigma)^{*} O(\tau) A O(\tau)^{*}\right)-9+\frac{9^{2}}{10} \operatorname{Tr}\left(O(\sigma) B O(\sigma)^{*} O(\tau) B O(\tau)^{*}\right)$

In particular, we may treat $\left.\partial_{t} \partial_{t}\right|_{t=0} f(t)$ as a symmetric 2 -form $\Omega(\cdot, \cdot)$ on the space of signed measures. 
In fact, the function $q$ is equivariant, so $q(\sigma, \tau)$ can be written as $g\left(\sigma^{-1} \tau\right):=$ $q\left(1, \sigma^{-1} \tau\right)$ and one can easily check that $g(\sigma)=g\left(\sigma^{-1}\right)$.

Three representative graphs of $g(\sigma)$ over two-parameter subspaces spanned by (respectively) $e_{1}$ and $e_{2}, e_{3}$ and $e_{4}$, and $e_{5}$ and $e_{6}$, are given below. We see that the function is mostly negative but there are smaller positive regions as well.

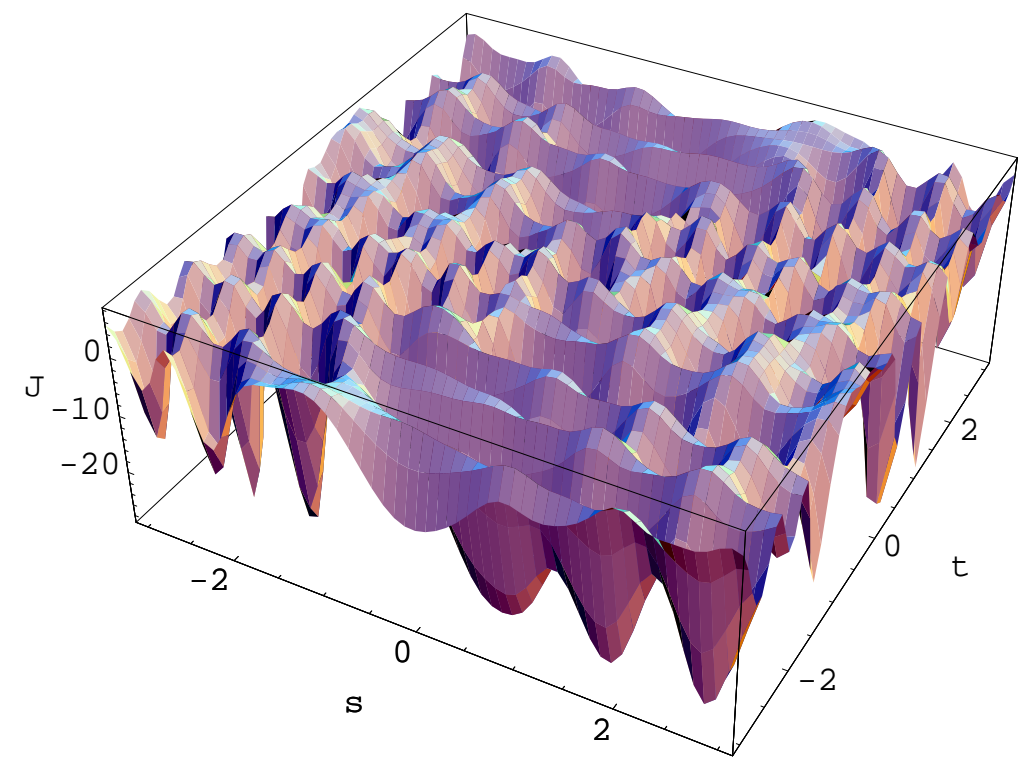

Figure 5: Graph of $g\left(s e_{1}+t e_{2}\right)$

Now we can take $\mu_{1}$ to be the sum of the atomic measures at the 55 points of $\partial_{F} X$ corresponding to the following rational parameters in $D$. Namely the points are of the form $K_{i} \cdot b^{+}$where $b^{+}$is the barycenter of a set of positive roots (note that any two choices will produce equivalent measures differing by a push forward by an isometry in the Weyl group) and that $K_{i}$ is the element of $K \approx S O(4)$ acting on $b^{+}$by conjugation corresponding to the element

$$
\exp \left(\begin{array}{cccc}
0 & s 1 & s 4 & s 6 \\
-s 1 & 0 & s 2 & s 5 \\
-s 4 & -s 2 & 0 & s 3 \\
-s 6 & -s 5 & -s 3 & 0
\end{array}\right) \in S O(4)
$$

For example, one such choice for $b^{+}$has coordinates $(2 / \sqrt{5}, 1 / \sqrt{5}, 0)$ in the 


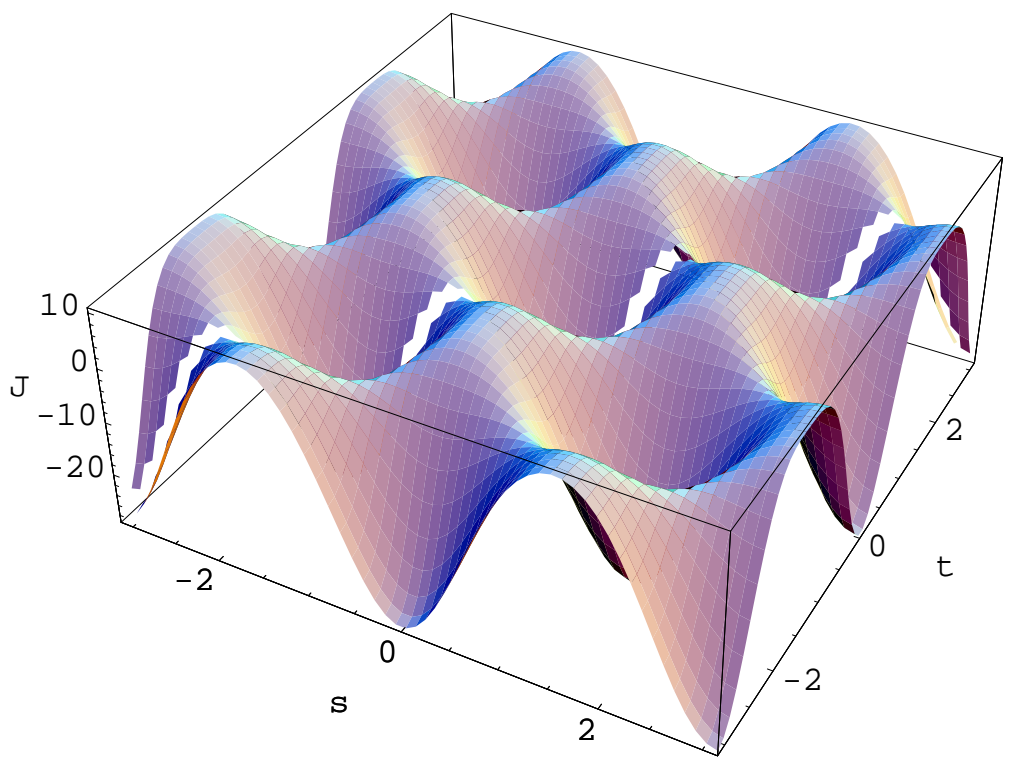

Figure 6: Graph of $g\left(s e_{3}+t e_{4}\right)$

canonical flat with the most common choice of coordinates in $S L(4)$. The corresponding values of the $s_{1}, \ldots, s_{6}$ for $K_{i}$ may be read across the $i$-th row from the following table:

$\begin{array}{cccccc}(-2.1763 & -1.1507 & -2.04369 & 0.44192 & 0.348006 & -0.0873793) \\ (1.10122 & -1.30303 & -1.07559 & 1.69668 & -0.537171 & -0.790495) \\ (-2.66081 & -2.51729 & 0.874064 & 0.0170786 & 2.76331 & 0.918491) \\ (3.06666 & -1.20383 & 3.10876 & -1.8274 & 1.33181 & 1.36971) \\ (2.14346 & 2.46489 & 0.233902 & -2.2138 & -1.34613 & -0.589322) \\ (2.27427 & 2.23082 & 2.87105 & 0.855587 & -0.33015 & -0.120273) \\ (2.39027 & 0.231281 & 1.93738 & 3.00424 & 2.76855 & 2.45438) \\ (2.01231 & 1.06647 & 2.80138 & 1.14019 & -2.46108 & 2.83835) \\ (-2.48367 & 1.81689 & 0.446606 & 1.91056 & 2.00405 & -0.735379) \\ (1.31393 & 2.82133 & 2.2746 & 1.55063 & -1.49752 & -0.199991) \\ (3.02592 & -1.82225 & -0.293302 & -0.0626386 & -2.88422 & -1.13504) \\ (0.835977 & 2.01248 & -2.54401 & 0.866367 & 0.15547 & 2.31572) \\ (3.08126 & 2.19107 & 2.85046 & -2.73643 & -2.06439 & -0.215145) \\ (-1.60506 & -2.41617 & -1.19739 & 1.37582 & 3.03405 & 0.925416) \\ (-1.08172 & 0.0564745 & 0.185755 & -2.15354 & -1.3391 & -1.95008) \\ (2.49137 & -1.02443 & -1.93669 & 0.325143 & -0.805692 & -0.198555) \\ (-1.87635 & 1.27567 & -0.514556 & -0.603716 & -2.95356 & -1.65078) \\ (-2.05109 & -1.32914 & 1.38543 & 0.114991 & -1.94354 & 0.887035) \\ (-0.674438 & -3.08308 & 1.0123 & -0.10102 & -2.47693 & 2.0086) \\ (1.66252 & -2.21819 & 2.60135 & -1.45814 & -0.67338 & 1.12196) \\ (1.33611 & 0.407789 & 2.98277 & -1.41592 & 1.14807 & -1.08302) \\ (1.89226 & 3.05482 & 2.90424 & 1.94358 & 0.694209 & -0.973808) \\ (0.437082 & 1.88506 & 2.8235 & 2.26881 & -0.227581 & 3.01805) \\ (-1.98061 & 1.3454 & 0.31266 & 1.3346 & 1.83436 & -2.91815) \\ (2.11814 & -2.21478 & 1.99318 & 1.63935 & -2.17152 & 2.00983) \\ (-3.04067 & 1.72613 & -1.93417 & -3.07534 & -0.593287 & -0.441659)\end{array}$




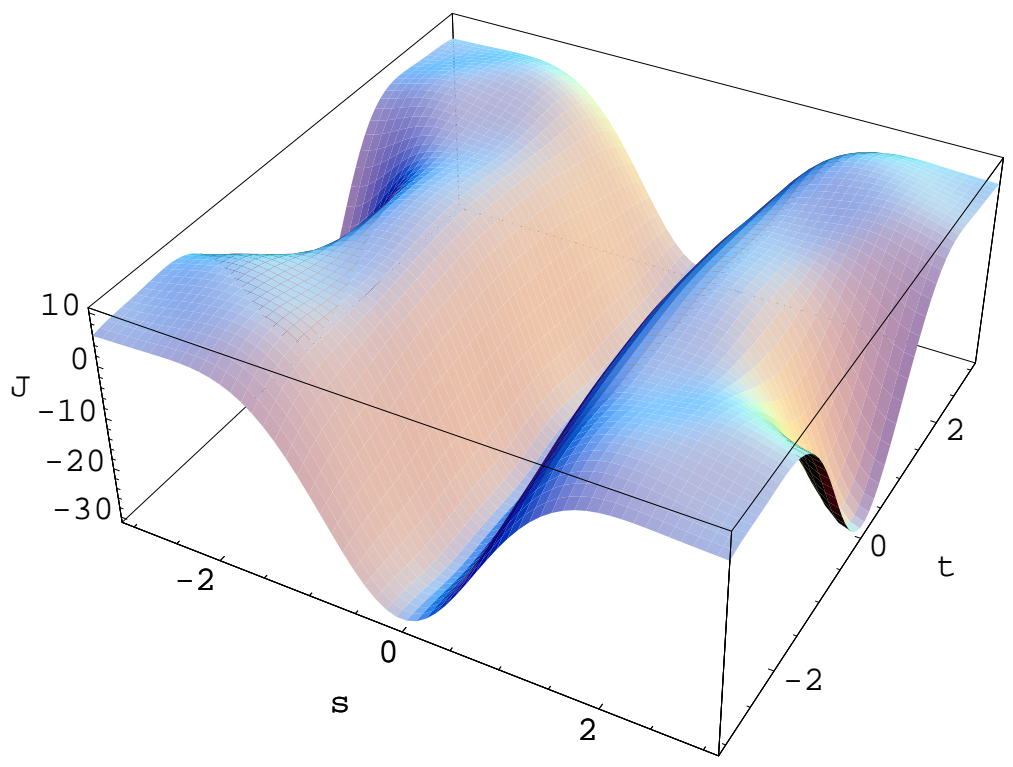

Figure 7: Graph of $g\left(s e_{5}+t e_{6}\right)$

$\begin{array}{cccccc}(0.770342 & -1.81881 & -0.275197 & 0.431129 & -2.14367 & -1.69527) \\ (-1.43618 & 2.22732 & 0.685263 & 0.111728 & -0.128947 & 2.00388) \\ (1.70871 & -0.815082 & 1.01946 & -2.77706 & 0.738644 & 0.316677) \\ (0.918539 & -1.36159 & -0.46878 & 0.250422 & -1.62977 & 2.22166) \\ (1.90247 & -1.07237 & 1.78702 & -1.35106 & 0.904547 & -2.51869) \\ (0.0816078 & -0.436794 & -2.92231 & 0.511173 & -2.93104 & 0.700914) \\ (-1.48943 & -1.81534 & -0.808906 & 0.336383 & 0.913519 & 1.00958) \\ (1.41415 & -1.44362 & -1.75929 & -2.38244 & -0.0976769 & -0.52368) \\ (-0.520171 & 1.83152 & 1.25689 & -2.31421 & 1.71687 & 1.20862) \\ (-1.96631 & 1.26418 & 1.49759 & -2.44414 & -2.17686 & -2.57833) \\ (-0.154573 & 2.51279 & 1.77364 & 0.226881 & 2.0735 & -1.63838) \\ (-2.7821 & -1.4711 & 0.6912 & -2.39754 & 0.457165 & 2.19418) \\ (-1.93022 & -1.08747 & 2.34186 & 1.36679 & -0.505503 & 0.8455) \\ (1.16658 & -3.03898 & 1.1385 & 0.148052 & 0.201847 & 2.68094) \\ (-1.84852 & 0.776858 & 1.5698 & -0.687528 & -0.780427 & -0.72635) \\ (1.21031 & -2.35802 & 1.66997 & -1.4704 & -2.38844 & -1.41061) \\ (0.458594 & 2.75866 & -1.58872 & 0.364191 & -2.1775 & -1.22843) \\ (0.386299 & 0.261576 & -0.174403 & 1.76511 & -2.95714 & 0.722224) \\ (-1.46748 & -2.15334 & -1.38535 & -1.73184 & 2.45454 & 1.7146) \\ (0.545927 & -2.51541 & -2.35701 & 0.0434106 & -0.207223 & 2.03679) \\ (0.325985 & 0.426342 & -1.7601 & -1.46899 & -0.638112 & -1.48682) \\ (0.995193 & 1.41102 & 2.67788 & -0.110336 & 0.810741 & -2.45279) \\ (1.00377 & -1.09859 & -0.9455 & 2.42064 & 1.69081 & 0.328404) \\ (1.65017 & 1.79446 & 0.906236 & -2.8566 & -1.2842 & 2.89926) \\ (-2.56134 & -0.141348 & -2.6657 & 1.22666 & 1.21836 & -1.79612) \\ (-0.519297 & 2.95723 & 1.68207 & 1.45581 & 1.81155 & 2.26842) \\ (-2.46329 & -0.587199 & -0.384539 & 2.98937 & -1.01251 & 2.22599) \\ (1.10689 & -1.94668 & 1.22285 & 1.941 & -0.7505 & -1.70434) \\ (0.642599 & -1.05925 & -1.2264 & 0.210594 & 2.56583 & -2.40472) \\ & & & & & \end{array}$

Each of the 55 atoms is weighted by the one of the three following corre- 
sponding vector of weights:

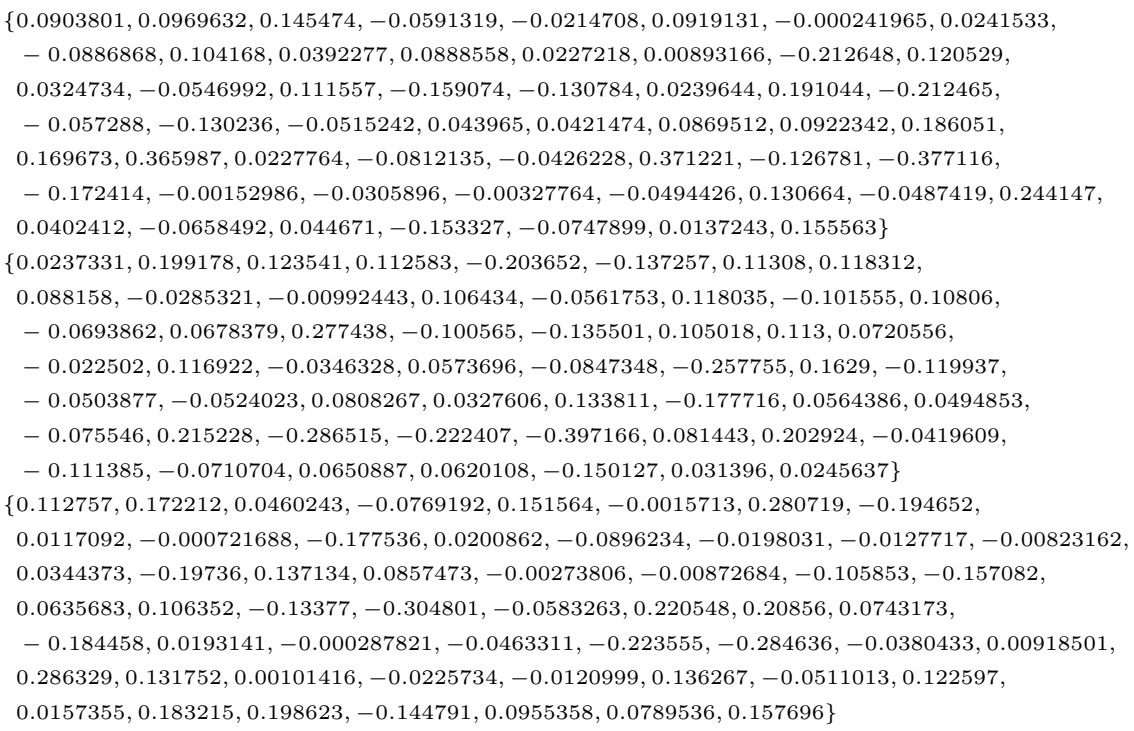

For $\mu_{1}$ chosen to be any of these three weighted sums of atomic measures on $\partial_{F} X$ it is not difficult (once the representation for $K$ has been computed) to show that the barycenter is $p$ in each case (the vector sum in $T_{p} X$ is 0 ) and that $\Omega\left(\mu_{1}, \mu_{1}\right)$ is greater than $1.13346,0.807823$, or 1.00141 corresponding to the three sets of weights above.

The only problem is that $\mu_{1}$ is not a positive measure since each of the above systems of weights has negative values. Nevertheless, we can fix this by adding elements in the kernel of $\Omega$. For any $\epsilon>0$ we may take a smooth (with respect to $\mu_{0}$ ) and symmetric (keeping barycenter 0) approximation $\mu_{1}^{\epsilon}$ to $\mu_{1}$ such that

$$
\left|\Omega\left(\mu_{1}, \mu_{1}\right)-\Omega\left(\mu_{1}^{\epsilon}, \mu_{1}^{\epsilon}\right)\right|<\epsilon
$$

Then if $c(\epsilon)=\min \left\{\inf \frac{d \mu_{1}^{\epsilon}}{\mu_{0}}, 0\right\}$, the measure

$$
\mu_{2}=\frac{-c(\epsilon) \mu_{0}+\mu_{1}^{\epsilon}}{|c(\epsilon)|+\left\|\mu_{1}^{\epsilon}\right\|}
$$

is a positive probability measure with barycenter $p$. Observe that

$$
\int_{D} O(\sigma) A O(\sigma)^{*} d \mu_{0}(\sigma)=\frac{1}{9} \mathrm{Id}
$$

and that

$$
\int_{D} O(\sigma) B O(\sigma)^{*} d \mu_{0}(\sigma)=\frac{\sqrt{20}}{9} \mathrm{Id}
$$


It follows from integrating inside the traces of the function $g\left(\sigma^{-1} \tau\right)$ that $\Omega\left(\mu_{0}, \mu\right)=0$ for any measure $\mu$. Therefore,

$$
\begin{aligned}
\Omega\left(\mu_{2}, \mu_{2}\right) & =\frac{1}{\left(|c(\epsilon)|+\left\|\mu_{1}^{\epsilon}\right\|\right)^{2}} \Omega\left(\mu_{1}^{\epsilon}, \mu_{1}^{\epsilon}\right) \\
& \geq \frac{1}{\left(|c(\epsilon)|+\left\|\mu_{1}^{\epsilon}\right\|\right)^{2}}(0.807823-\epsilon)
\end{aligned}
$$

which is positive for $\epsilon<0.8$.

The consequence of this is that $f(t)$ is larger than 1 for some sufficiently small $t$, and $\mu=\mu_{2}$. Hence there is no iso-derivative type inequality for the Jacobian among (positive) probability measures which is sharp at $\mu_{0}$.

In actuality the Jacobian at $\frac{\mu_{0}+t \mu_{2}}{\left\|\mu_{0}+t \mu_{2}\right\|}$ stays less than 1.01, and probably

much less, because $\frac{1}{\left(|c(\epsilon)|+\left\|\mu_{1}^{\epsilon}\right\|\right)^{2}}$ decays at least quadratically in $\epsilon$ as $\epsilon \longrightarrow 0$ for any choice of $\mu_{1}^{\epsilon}$.

\section{References}

[Al] P. Albuquerque, Patterson-Sullivan theory in higher rank symmetric spaces, Geom. Funct. Anal. (GAFA), Vol. 9 (1999), 1-28.

[BGS] W. Ballmann, M. Gromov and V. Schroeder, Manifolds of nonpositive curvature, Progress in Mathematics, Vol. 61, Birkaüser, 1985.

[BaCS] D. Bao, S.S. Chern and Z. Shen, An introduction to RiemannFinsler geometry, Graduate Texts in Mathematics, 200. Springer-Verlag, New York, 2000.

[Be1] L. Bessières, Un theoreme de rigidite differentielle, Comment. Math. Helv. 73 (1998), No.3, 443-479.

[Be2] Sur le volume minimal des varits ouvertes, Ann. Inst. Fourier (Grenoble) 50 (2000), no. 3, 965-980.

[BCG1] G. Besson, G. Courtois and S. Gallot, Entropies et rigidités des espaces localement symétriques de courbure strictement négative, Geom. Funct. Anal. 5 (1995), no. 5, 731-799.

[BCG2] _ Minimal entropy and Mostow's rigidity theorems, Ergodic Theory Dynam. Sys. 16 (1996), no. 4, 623-649. 
[BCG3] _ Volumes and rigidities of Riemannian manifolds, Lecture Notes, Korean Advanced Institute for Sciences and Technlogy, July 1998.

[BCG4]_, A real Schwarz lemma and some applications. Rend. Mat. Appl., VII. Ser. 18, No.2, 381-410 (1998).

[BCG5] L Lemme de Schwarz rel et applications gomtriques, Acta Math. 183 (1999), no. 2, 145-169.

[BC] J. Boland and C. Connell, Entropy rigidity for foliations of compact spaces, To appear in Israel J. Math.

[BCS] J. Boland, C. Connell and J. Souto, Minimal entropy rigidity for finite volume manifolds, Preprint (1999).

[BN] J. Boland and F. Newberger, Minimal entropy rigidity for Finsler manifolds of negative flag curvature. Ergodic Theory Dynam. Systems 21 (2001), no. 1, 13-23.

[Bon] F. Bonahon A Schläfli-type formula for convex cores of hyperbolic 3-manifolds, J. Differential Geom. 50 (1998), 25-58.

[BGP] Y. Burago, M. Gromov and G.A. Perel'man, A. D. Aleksandrov spaces with curvatures bounded below, Uspekhi Mat. Nauk 47 (1992), no. 2(284), 3-51, 222; translation in Russian Math. Surveys 47 (1992), no. 2, 1-58.

[BP] K. Burns and G.P. Paternain, Anosov magnetic flows, critical values and topological entropy, Preprint (2001).

[CMT] R. Canary, Y. Minsky and E. Taylor, Spectral theory, Hausdorff dimension and the topology of hyperbolic 3-manifolds, Journal of Geometric Analysis 9 (1999), 17-40.

[CG] J. Cheeger and M. Gromov, Chopping Riemannian manifolds, Differential geometry, 85-94, Pitman Monogr. Surveys Pure Appl. Math. 52, Longman Sci. Tech., Harlow, 1991.

[CF1] C. Connell and B. Farb, Minimal entropy rigidity for lattices in products of rank one symmetric spaces, Comm. in Analysis and Geometry, to appear.

[CF2] _ The Degree Theorem in higher rank, November 2000 preprint. 
$[\mathrm{CHK}]$ D. Cooper, C.D. Hodgson and S.P. Kerckhoff, Threedimensional orbifolds and cone-manifolds, With a postface by Sadayoshi Kojima. MSJ Memoirs 5, Mathematical Society of Japan, Tokyo, 2000.

[Eb] P. Eberlein, Geometry of nonpositively curved manifolds, Chicago Lectures in Math. Univ. of Chicago Press, 1996.

[FJ] F.T. Farrell and L.E. Jones, Topological rigidity for compact nonpositively curved manifolds, Differential geometry: Riemannian geometry (Los Angeles, CA, 1990), 229-274, Proc. Sympos. Pure Math. 54, Part 3, Amer. Math. Soc., Providence, RI, 1993.

[Fl] L. Flaminio, Local entropy rigidity for hyperbolic manifolds, Comm. Anal. Geom. 3 (1995), no. 3-4, 555-596.

[Ga] S. Gallot, Curvature-decreasing maps are volume-decreasing (on joint work with G. Besson and G. Courtois), Proc. ICM, Vol. II (Berlin, 1998), Doc. Math. 1998, 339-348.

[Gr] M. Gromov, Volume and bounded cohomology, Inst. Hautes Études Sci. Publ. Math. 56 (1983), 5-99.

[Gr2] Filling Riemannian manifolds, J. Diff. Geom. 18 (1983), 1-147.

[Hu] S. Hurder, Coarse geometry of foliations. Geometric study of foliations, 35-96, World Sci. Publishing, River Edge, NJ, 1994.

[Ka] A. Katok, Entropy and closed geodesics, Ergodic Theory Dynam. Systems 2 (1982), no. 3-4, 339-365.

[Kn] G. Knieper, On the asymptotic geometry of nonpositively curved manifolds, Geom. Funct. Anal. (GAFA) 7 (1997), 755-782.

[Ma] A. Manning, Topological entropy for geodesic flows, Annals of Math. 110 (1979), 567-573.

[Pa] P. Pansu, Volume, courbure et entropie (d'après G. Besson, G. Courtois et S. Gallot), Séminaire Bourbaki, Vol. 1996-97, Astérisque No. 245 (1997), Exp. No. 823, 3, 83-103. 
[PaPa1] G.P. Paternain and M. Paternain, Anosov geodesic flows and twisted symplectic structures, International Conference on Dynamical Systems (Montevideo, 1995), Pitman Res. Notes Math. Ser., 362 (1996), 132-145.

[PaPa2] First derivative of topological entropy for Anosov geodesic flows in the presence of magnetic fields, Nonlinearity 10 (1997), no. 1, 121-131.

[PaPe] G.P. Paternain and J. Petean, Minimal entropy and collapsing with curvature bounded from below, Preprint (2001).

[Pr] G. Prasad, Discrete subgroups isomorphic to lattices in semisimple Lie groups, Amer. J. Math. 98 (1976), no. 1, 241-261.

[Sam1] A. Sambusetti, Einstein manifolds, volume rigidity and SeibergWitten theory, Sémin. Théor. Spectr. Géom. Année 1998-1999, Univ. Grenoble I, Saint-Martin-d'Héres 17 (1999), 163-184.

[Sam2] Minimal entropy and simplicial volume, Manuscripta Math. 99 (1999), no. 4, 541-560.

[Sa] R. Savage, The space of positive definite matrices and Gromov's invariant, Trans. Amer. Math. Soc. 274 (1982), no. 1, 239-263.

[St] P. Storm, Minimal volume Alexandrov spaces, Preprint (2001).

[Ta] E. Taylor, Geometric finiteness and the convergence of Kleinian groups, Communications in Analysis and Geometry 5 (1997), no. $3,497-533$.

[Th1] W. Thurston, The topology and geometry of 3-manifolds, Princeton Univ., Lecture Notes (1976-79).

[Th2] , Hyperbolic geometry and 3-manifolds, in Lowdimensional topology (Bangor, 1979), London Math. Soc. Lecture Note Ser., 48, Cambridge Univ. Press, Cambridge-New York (1982), 9-25.

[Ush] A. Ushijima, The canonical decompositions of some family of compact orientable hyperbolic 3-manifolds with totally geodesic boundary, Geometriae Dedicata 78 (1999), 21-47. 
[Ve] P. Verovic, Problème de l'entropie minimale pour les métriques de Finsler, Erg. Theo. and Dynam. Syst., 19 (1999), no. 6, $1637-1654$.

[Zi] R. Zimmer, Ergodic theory, semisimple Lie groups, and foliations by manifolds of negative curvature, Inst. Hautes tudes Sci. Publ. Math. 55 (1982), 37-62.

Dept. of Mathematics, University of Chicago

5734 University Ave.

Chicago, Il 60637

E-mail: cconnell@math.uic.edu,farb@math.uchicago.edu 